\title{
The Exponential T-X Family of Distributions: Properties and an Application to Insurance Data
}

\author{
Zubair Ahmad (D), ${ }^{1}$ Eisa Mahmoudi $\left(D,{ }^{1}\right.$ Morad Alizadeh $\left(\mathbb{D},{ }^{2}\right.$ Rasool Roozegar $(D)$, \\ and Ahmed Z. Afify $\mathbb{D}^{3}$ \\ ${ }^{1}$ Department of Statistics, Yazd University, P.O. Box 89175-741, Yazd, Iran \\ ${ }^{2}$ Department of Statistics, Persian Gulf University, Bushehr 75169, Iran \\ ${ }^{3}$ Department of Statistics, Mathematics and Insurance, Benha University, Benha 13511, Egypt
}

Correspondence should be addressed to Eisa Mahmoudi; emahmoudi@yazd.ac.ir

Received 18 July 2020; Revised 31 March 2021; Accepted 21 April 2021; Published 5 May 2021

Academic Editor: Markos Koutras

Copyright ( 92021 Zubair Ahmad et al. This is an open access article distributed under the Creative Commons Attribution License, which permits unrestricted use, distribution, and reproduction in any medium, provided the original work is properly cited.

Heavy-tailed distributions play a prominent role in actuarial and financial sciences. In this paper, we introduce a family of distributions that we refer to as exponential T-X (ETX) family. Based on the proposed approach, a new extension of the Weibull model is introduced. The proposed model is very flexible in modeling heavy-tailed data. Some mathematical properties are derived, and maximum likelihood estimates of the model parameters are obtained. A Monte Carlo simulation study is conducted to evaluate the performance of the maximum likelihood estimators. Actuarial measures such as value at risk and tail value at risk are also calculated. A simulation study based on these actuarial measures is provided. Finally, an application to a heavy-tailed automobile insurance claim data set is presented. The proposed model is compared with some well-known competing distributions.

\section{Introduction}

Broadly speaking, statistical distributions play a prominent role in modeling data in applied fields, particularly in risk management, economic, financial, and actuarial sciences. However, the quality of the procedures primarily depends upon the assumed probability model of the phenomenon under consideration. Among the applied fields, insurance losses are usually positive, right-skewed, unimodal, and with heavy tails; see the works of Lane [1], Cooray and Ananda [2], Klugman et al. [3], and Ahmad et al. [4]. The actuaries are often looking for heavytailed distributions to sufficiently provide a good estimate of the associated business risk level. The heavy-tailed distributions are those whose right tail probabilities are heavier than the exponential one, and they satisfy

$$
\lim _{x \rightarrow \infty} e^{p x}[1-G(x)]=\infty, \quad p>0 .
$$

An important characteristic of the heavy-tailed distributions is the regular variational property. A distribution is called regular varying if it obeys

$$
\lim _{x \longrightarrow \infty} \frac{1-G(t x)}{1-G(x)}=t^{-a}, \quad a \in\{0, \infty\},
$$

where $a$ is a so-called index of regular variation. The distributions that possess the regular variation property are very competitive models for modeling heavy-tailed data sets. For more details, the interested readers can refer to the works of McNeil [5] and Beirlant et al. [6].

Well-known probability models such as the log-normal, Pareto, gamma, beta, and Weibull distributions are very useful in modeling data in application. However, these classical distributions have certain deficiencies in modeling insurance losses. For example, (i) the log-normal, gamma, and beta distributions do not have closed-form expressions for the cumulative distribution function (cdf) and the computation of many mathematical properties becomes difficult, (ii) the Pareto distribution, due to the monotonically decreasing shape of the density, does not provide a reasonably good fit for many applications, and (iii) the Weibull distribution covers better the behavior of small losses but fails to cater the behavior of large losses [7]. 
Therefore, the practitioners have shown a deep interest in proposing extended versions of these existing distributions. The new developments have been constructed through many approaches such as (i) composition of two or more distributions, (ii) compounding of distributions, and (iii) a finite mixture of distributions (Scollnik and Sun [8] and Ahmad et al. [9]).

The composition of two or more distributions is a prominent approach for obtaining new flexible heavy-tailed families of distributions, which gives a reasonably good fit for heavy-tailed losses as shown by Cooray and Ananda [2], Nadarajah and Abu Bakar [10], and Abu Bakar et al. [11]. However, it should be noted that the new distributions obtained by the composition approach involve more than three parameters causing difficulties in the estimation process and computational efforts are required.

Another prominent approach is the compounding of distributions to cater the data modeling with unimodality, right-skewness, and heavy tails as illustrated by Punzo [12], Mazza and Punzo [13], Tomarchio and Punzo [14], and Punzo and Bagnato [15]. However, the density obtained via this method may not have a closed-form expression, which makes the estimation more cumbersome as shown in Punzo et al. [16]. For a brief review about compounding of distributions, we refer to the work of Tahir and Cordeiro [17].

Finite mixture models represent a further approach to define very flexible distributions which are also able to capture, for instance, multimodality of the underlying distribution as shown in the works of Bernardi et al. [18], Miljkovic and Grun [19], and Punzo et al. [20]. The price to pay for this greater flexibility is a more complicated and computationally challenging inference.

In this article, we introduce a family of distributions called exponential T-X (ETX) family of distributions. The proposed method possesses the regularly tail behavior and therefore it can be used quite effectively for modeling heavytailed data. Using the ETX method, we study a special model named exponential T-X Weibull (ETX-Weibull) distribution. We later prove empirically that the ETX-Weibull distribution provides better fits than the well-known competitive distributions in terms of different measures of model validation using automobile insurance claim data.

We hope that the ETX-Weibull distribution will attract wider applications in insurance losses data and financial returns, among others. The estimation of the ETX-Weibull distribution parameters using the method of maximum likelihood estimation has been carried out. Further, some actuarial measures such as value at risk ( $\mathrm{VaR})$ and tail value at risk (TVaR) are also calculated.

The rest of this article is organized in six sections as follows: the methodology and ETX-Weibull model are discussed in Section 2. The maximum likelihood estimators of the ETX-Weibull parameters are obtained in Section 3. A Monte Carlo simulation study is provided in Section 4. The actuarial measures of the ETX-Weibull distribution and their simulation study are derived in Section 5. A practical application to the automobile insurance claims data set is provided in Section 6. Finally, the article is concluded in the last section.

\section{Methodology, Special Model, and Properties}

This section deals with the methodology adopted to introduce the proposed method and a special model of the proposed model. Furthermore, the regular variation results and other mathematical properties are also derived.

2.1. Methodology. In this subsection, we introduce a new family of distributions using the T- $X$ family approach [21]. Let $v(t)$ be the probability density function (pdf) of a random variable, say $T$, where $T \in\left[a_{1}, a_{2}\right]$ for $-\infty \leq a_{1}<a_{2} \leq \infty$ and let $W[F(x ; \xi)]$ be a function of cumulative distribution function (cdf) of a random variable, say $X$, depending on the parameter vector $\xi$ and satisfying the conditions given below:

(1) $W[F(x ; \xi)] \in\left[a_{1}, a_{2}\right]$

(2) $W[F(x ; \xi)]$ is differentiable and monotonically increasing

(3) $W[F(x ; \xi)] \longrightarrow a_{1} \quad$ as $\quad x \longrightarrow-\infty \quad$ and $W[F(x ; \xi)] \longrightarrow a_{2}$ as $x \longrightarrow \infty$

The $\mathrm{T}-X$ family method is defined by

$$
G(x)=\int_{a_{1}}^{W[F(x ; \xi)]} v(t) \mathrm{d} t, \quad x \in \mathbb{R},
$$

where $W[F(x ; \xi)]$ satisfies the conditions stated above. The pdf corresponding to (3) is

$$
g(x)=\left\{\frac{\partial}{\partial x} W[F(x ; \xi)]\right\} v\{W[F(x ; \xi)]\}, \quad x \in \mathbb{R}
$$

For the contributed work on T- $X$ method, we refer to the work of Ahmad et al. By taking $v(t)$ as the pdf of the exponential distribution with rate parameter $(\theta=1)$ given by $v(t)=e^{-t}$ and replacing the upper limit of (3) with $W[F(x ; \xi)]=-\log \{\sigma \bar{F}(x ; \xi) / \sigma-F(x ; \xi)\}$, we get the cdf of the proposed family. If a random variable $X$ follows one of the members of our ETX family, then its cdf is given by

$$
G(x ; \sigma, \xi)=1-\frac{\sigma \bar{F}(x ; \xi)}{\sigma-F(x ; \xi)}, \quad \sigma>1, \xi>0, x \in \mathbb{R},
$$

where $\bar{F}(x ; \xi)=1-F(x ; \xi)$ is the survival function (sf) of the baseline random variable depending on the parameter $\xi$.

To the best of our knowledge, the proposed method has not been used so far. This is another motivation using the proposed approach. Hence, using the proposed method a number of new distributions can also be obtained. The probability density function (pdf) corresponding to (5) is given by

$$
g(x ; \sigma, \xi)=\frac{\sigma(\sigma-1) f(x ; \xi)}{(\sigma-F(x ; \xi))^{2}}, \quad x \in \mathbb{R} .
$$

The new pdf is most tractable when $F(x ; \xi)$ and $f(x ; \xi)$ have simple analytical expressions. The basic motivations for using the ETX family of distributions in practice are the following: 
(1) A new prominent method of introducing an additional parameter to generate generalized versions of the baseline model rather than adding two or more parameters

(2) To improve the characteristics and flexibility of the classical distributions

(3) To make the kurtosis more flexible as compared to the baseline model

(4) To obtain new models suitable for modeling heavytailed data

(5) To define special models having closed forms for cdf and sf as well as thefailure rate function

(6) To provide consistently better fits than other generated distributions having the same or higher number of parameters

The survival function (sf) and hazard rate function (hrf) corresponding to (5) are given, respectively, by

$$
\bar{G}(x ; \sigma, \xi)=\frac{\sigma \bar{F}(x ; \xi)}{\sigma-F(x ; \xi)}, \quad x \in \mathbb{R},
$$

and

$$
h(x ; \sigma, \xi)=\frac{(\sigma-1) f(x ; \xi)}{\bar{F}(x ; \xi)(\sigma-F(x ; \xi))}, \quad x \in \mathbb{R} .
$$

Based on (5), we propose a three-parameter special submodel, called ETX-Weibull distribution. We derive explicit expressions for the $\mathrm{VaR}$ and $\mathrm{TVaR}$ of the proposed distribution. Most importantly, we provide a comprehensive simulation study of the VaR and TVaR and empirically show that the ETX-Weibull distribution is a heavy-tailed model and can be used quite effectively in the field of insurance sciences and other related areas.

2.2. Special Model. Consider the distribution and density functions of the two-parameter Weibull distribution given by $F(x ; \xi)=1-e^{-\gamma x^{\alpha}}, x \geq 0$ and $f(x ; \xi)=\alpha \gamma x^{\alpha-1} e^{-\gamma x^{\alpha}}$, respectively, where $\xi=(\alpha, \gamma)$. Then, the cdf of the ETXWeibull distribution is given by

$$
G(x ; \sigma, \xi)=1-\frac{\sigma e^{-\gamma x^{\alpha}}}{\sigma+e^{-\gamma x^{\alpha}}-1}, \quad x \geq 0, \alpha, \gamma>0, \sigma>1 .
$$

with pdf

$$
g(x ; \sigma, \xi)=\frac{\alpha \gamma \sigma(\sigma-1) x^{\alpha-1} e^{-\gamma x^{\alpha}}}{\left(\sigma+e^{-\gamma x^{\alpha}}-1\right)^{2}}, \quad x>0 .
$$

The effect of the parameter $\sigma$ on the shapes of pdf of the proposed model are shown in Figure 1. These density plots are presented for $\alpha=1.2, \gamma=0.5$, and different values of $\sigma$. From Figure 1, it is clear that the proposed model tends to a heavy-tailed distribution as the value of $\sigma$ increases. These plots illustrate that the additional parameter $\sigma$ has a significant effect on the pdf behavior of the proposed model.

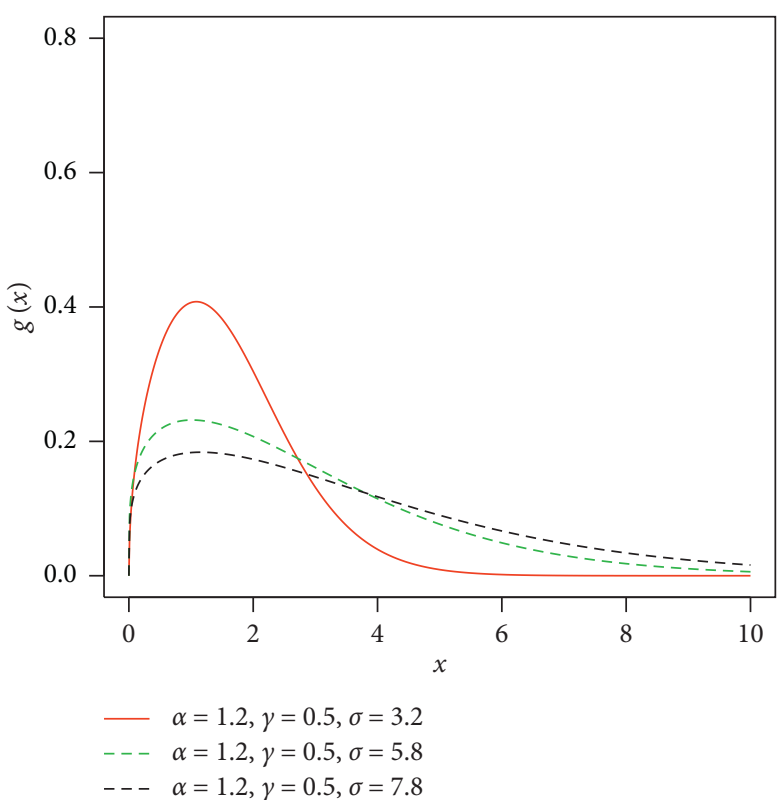

Figure 1: Pdf plots of the ETX-Weibull model for $\alpha=1.2, \gamma=0.5$, and different values of $\sigma$.

2.3. Mathematical Properties. In this section, some mathematical properties of the proposed family such as regularly varying tail behavior, quantile function, moments, and moment generating function are derived.

2.3.1. Regularly Varying Tail Behavior. The regularly varying tail behavior is an important characteristic to identify heavytailed distributions. In this subsection, we deal with the regular variational behavior of the proposed family. According to [22], in terms of sf, we have the following characterization.

Theorem 1. If $\bar{F}(x ; \xi)$ is regularly varying, then so is $\bar{G}(x ; \sigma, \xi)$.

Proof. Assume that $\lim _{x \rightarrow \infty} \bar{F}(a x) / \bar{F}(x)=g(a)$ is finite but nonzero for every $a>0$. Using expression 7 , we observe that

$$
\frac{\bar{G}(a x ; \sigma, \xi)}{\bar{G}(x ; \sigma, \xi)}=\frac{\sigma \bar{F}(a x ; \xi)}{\sigma \bar{F}(x ; \xi)} \cdot \frac{\sigma-F(x ; \xi)}{\sigma-F(a x ; \xi)} .
$$
to

Since $\lim _{x \longrightarrow \infty} F(x ; \xi)=1$, the expression in (11) reduces

$$
\begin{aligned}
& \lim _{x \rightarrow \infty} \frac{\bar{G}(a x)}{\bar{G}(x)}=g(a) \cdot \frac{\sigma-1}{\sigma-1}, \\
& \lim _{x \rightarrow \infty} \frac{\bar{G}(a x)}{\bar{G}(x)}=g(a),
\end{aligned}
$$

which is finite but nonzero for every $a>0$; thus, $\bar{G}(x, \sigma, \xi)$ is regularly varying. 
Remark 1. By Karamata's characterization theorem [23], the function $g$ has the form $g(a)=a^{\rho}$, where $\rho \in \mathbb{R}$ is the socalled index of regular variation, and $a>0$.

2.3.2. Regular Variational Result. If the distribution of $X$ has power law behavior, then, according to [22], we have

$$
\bar{F}(x)=\mathbb{P}(X>x) \sim x^{-\alpha} .
$$

Now, by Karamata's characterization theorem [23], this means that we should be able to write $\bar{G}(x ; \sigma, \xi)$ as

$$
\bar{G}(x ; \sigma, \xi)=x^{-\alpha} L(x),
$$

where $L(x)$ is slowly varying. Note that

$$
\bar{G}(x ; \sigma, \xi)=\frac{\sigma \bar{F}(x ; \xi)}{\sigma-1+\bar{F}(x ; \xi)} .
$$

Since $\bar{F}(x ; \xi) \sim x^{-\alpha}$, we can write

$$
\bar{G}(x ; \sigma, \xi)=\frac{\sigma x^{-\alpha}}{\sigma-1+x^{-\alpha}}=x^{-\alpha} L(x),
$$

where $L(x)=\sigma / \sigma-1+x^{-\alpha}$. So if $L(x)$ is slowly varying, then the variational result obtained is true. Now, according to Resnick [24], we must show that, for all $a>0$,

$$
\lim _{x \rightarrow \infty} \frac{L(a x)}{L(x)}=1 \text {. }
$$

After some simplification, we get

$$
\frac{L(a x)}{L(x)}=\frac{\sigma\left(\sigma-1+1 / x^{\alpha}\right)}{\sigma\left(\sigma-1+1 / x^{\alpha} a^{\alpha}\right)} .
$$

If $x \longrightarrow \infty$, then $\lim _{x \rightarrow \infty} 1 / x^{\alpha}=0, \quad$ and $\lim _{x \longrightarrow \infty} 1 / x^{\alpha} a^{\alpha}=0$. Therefore, from expression (18), we have

$$
\frac{L(a x)}{L(x)}=\frac{\sigma(\sigma-1)}{\sigma(\sigma-1)}
$$

which leads to the fact that

$$
\lim _{x \rightarrow \infty} \frac{L(a x)}{L(x)}=1
$$

2.3.3. The Quantile Function. The quantile function of distribution is very useful to generate random numbers by Monte Carlo simulation. The quantile function of a random variable $X$ with cdf (5) is given by

$$
x_{q}=Q(u)=G^{-1}(u)=F^{-1}(t),
$$

where $t$ is the solution of the equation $t(\sigma+u-1)-u \sigma=0$, and $u \in \in(0,1)$. The nonlinear expression (21) can be used to obtain the random numbers from any submodel of the proposed class.
2.3.4. Moments. Suppose that $X$ is a random variable with pdf (6); then the $r^{\text {th }}$ moment of the proposed family is derived as

$$
\mu_{r}^{\prime}=\int_{-\infty}^{\infty} x^{r} \frac{\sigma(\sigma-1) f(x ; \xi)}{(\sigma-F(x ; \xi))^{2}} \mathrm{~d} x
$$

Using the series, we have

$$
\frac{1}{(1-x)^{2}}=\sum_{n=0}^{\infty} n x^{n-1} \text {. }
$$

Replacing $x$ by $F(x ; \xi) / \sigma$ in (23) and then using it in (22), we arrive at

$$
\mu_{r}^{\prime}=\sum_{n=0}^{\infty} \frac{n(\sigma-1)}{\sigma^{n}} \eta_{r, n},
$$

where $\eta_{r, n}=\int_{-\infty}^{\infty} x^{r} f(x ; \xi) F(x ; \xi)^{n-1} \mathrm{~d} x$.

For $r=1,2,3,4$, we get the first four moments of the ETX family. The effects of the shape parameters on the skewness and kurtosis can be detected on the moments. The $r^{\text {th }}$ moment of the ETX-Weibull distribution can be calculated using (24) as follows. Using the pdf and cdf of the twoparameter Weibull distribution (defined in Section 2.2), $\eta_{r, n}$ can be expressed as

$$
\begin{aligned}
\eta_{r, n} & =\int_{0}^{\infty} x^{r} \alpha \gamma x^{\alpha-1} e^{-\gamma x^{\alpha}}\left(1-e^{-\gamma x^{\alpha}}\right)^{n-1} \mathrm{~d} x \\
& =\frac{1}{n} \int_{0}^{\infty} x^{r} h_{n}(x ; \alpha, \gamma) \mathrm{d} x,
\end{aligned}
$$

where $h_{n}(x ; \alpha, \gamma)$ represents the exponentiated Weibull (Exp-Weibull) density with the baseline Weibull (defined in Section 2.2) with power parameter $n>0$. Hence, $\eta_{r, n}$ reduces to

$$
\eta_{r, n}=\gamma^{-r / \alpha}\left(1+\frac{r}{\alpha}\right) \sum_{k=0}^{\infty} \varphi_{k}^{(n)},
$$

where $\varphi_{k}^{(n)}=(-1)^{k}(k+1)^{(-r / \alpha)-1}\left(\begin{array}{c}n-1 \\ k\end{array}\right)$. Using equations (24) and (26), the $r^{\text {th }}$ moment of the ETX-Weibull distribution reduces to

$$
\mu_{r}^{\prime}=\sum_{n, k=0}^{\infty} \varphi_{k}^{(n)} \frac{n(\sigma-1)}{\sigma^{n}} \gamma^{-r / \alpha}\left(1+\frac{r}{\alpha}\right) .
$$

The $s^{\text {th }}$ central moment of the ETX-Weibull distribution can be expressed as

$$
\mu_{s}=\sum_{j=0}^{s}(-1)^{s-j}\left(\begin{array}{l}
s \\
j
\end{array}\right) \mu_{1}^{{ }^{s}-j} \mu_{s}^{\prime} .
$$

where $\mu_{1}^{\prime}$ follows from (27) with $r=1$. Hence, $\mu_{s}$ of the ETXWeibull distribution takes the form 


$$
\mu_{s}=\sum_{j=0}^{s} \sum_{n, k=0}^{\infty}(-1)^{s-j}\left(\begin{array}{c}
s \\
j
\end{array}\right)\left[\varphi_{k}^{(n)} \frac{n(\sigma-1)}{\sigma^{n}} \gamma^{-1 / \alpha}\left(1+\frac{1}{\alpha}\right)\right]^{s-j} \times \varphi_{k}^{(n)} \frac{n(\sigma-1)}{\sigma^{n}} \gamma^{-s / \alpha}\left(1+\frac{s}{\alpha}\right)
$$

Hence, the second, third, and fourth moments, $\mu_{2}, \mu_{3}$, and $\mu_{4}$, of the ETX-Weibull distribution follow simply from (29) by replacing $s=2,3,4$, respectively. Based on these moments, we can obtain skewness and kurtosis measures of the ETX-Weibull distribution. The skewness and kurtosis of ETX-Weibull distribution are defined by

$$
\begin{gathered}
\text { Skewness }=\frac{\mu_{3}}{\mu_{2}^{3 / 2}}, \\
\text { Kurtosis }=\frac{\mu_{4}}{\mu_{2}^{2}} .
\end{gathered}
$$

These measures are less sensitive to outliers. Plots for the skewness and kurtosis of the ETX-Weibull distribution are displayed in Figure 2.
Furthermore, the moment generating function, say $M_{X}(t)$, of the ETX family of distributions can be obtained as follows:

$$
M_{X}(t)=\sum_{r, n=0}^{\infty} \frac{n(\sigma-1) t^{r}}{\sigma^{n} r !} \eta_{r, n}
$$

\section{Maximum Likelihood Estimation}

In the following section, we use the maximum likelihood estimation method to estimate the model parameters. Let $x_{1}, x_{2}, \ldots, x_{n}$ be the observed values of a random sample of size $n$ taken from pdf (6) with parameters $\sigma$ and $\xi$. Then, the log-likelihood function corresponding to (6) is given by

$$
L\left(x_{i} ; \sigma, \xi\right)=n \log \sigma+n \log (\sigma-1)+\sum_{i=1}^{n} \log f\left(x_{i} ; \xi\right)-2 \sum_{i=1}^{n} \log \left[\sigma-F\left(x_{i} ; \xi\right)\right]
$$

The log-likelihood function can be maximized either directly or by solving the nonlinear likelihood function obtained by differentiating (25). We used the goodness of fit function in $R$ with "L-BFGS-B" algorithm to obtain the MLEs. The partial derivatives of (25) with respective to parameters are given, respectively, by

$$
\begin{aligned}
& \frac{\partial}{\partial \sigma} L\left(x_{i} ; \sigma, \xi\right)=\frac{n}{\sigma}+\frac{n}{\sigma-1}-2 \sum_{i=1}^{n} \frac{1}{\sigma-F\left(x_{i} ; \xi\right)} \\
& \frac{\partial}{\partial \xi} L\left(x_{i} ; \sigma, \xi\right)=\sum_{i=1}^{n} \frac{\partial f\left(x_{i} ; \xi\right) / \partial \xi}{f\left(x_{i} ; \xi\right)}+2 \sum_{i=1}^{n} \frac{\partial F\left(x_{i} ; \xi\right) / \partial \xi}{\sigma-F\left(x_{i} ; \xi\right)}
\end{aligned}
$$

Solving numerically the above expressions simultaneously yields the MLEs (maximum likelihood estimators) $(\widehat{\sigma}, \widehat{\xi})$ of $(\sigma, \xi)$, respectively.

\section{Monte Carlo Simulation Study}

In this section, we assess the behavior of the maximum likelihood estimators for a finite sample of size $n$. Simulation study based on the ETX-Weibull distribution is carried out. The random numbers are generated via the quantile technique from the ETX-Weibull using optim() R-function with the argument method $=$ L-BFGS-B ; see Appendix. The simulation study is based on the following steps:

(1) We generate $N=1000$ samples of sizes $n=$ $25,50, \ldots, 1000$ from the ETX-Weibull distribution

(2) Compute the maximum likelihood estimates for the model parameters

(3) Compute the MSEs and biases given by

$$
\begin{aligned}
& \operatorname{MSE}(\boldsymbol{\Theta})=\frac{1}{1000} \sum_{i=1}^{1000}(\widehat{\boldsymbol{\Theta}}-\boldsymbol{\Theta})^{2} \\
& \operatorname{bias}(\boldsymbol{\Theta})=\frac{1}{1000} \sum_{i=1}^{1000}(\widehat{\boldsymbol{\Theta}}-\boldsymbol{\Theta})
\end{aligned}
$$

for $\Theta=(\alpha, \sigma, \gamma)$, respectively

The simulation results are graphically displayed in Figures 3-6.

From these plots, we observe the following results:

(i) The estimates tend to be stable as the sample size $n$ increases

(ii) The estimated MSEs decay toward zero as n increases

(iii) The absolute biases decrease as $n$ increases 


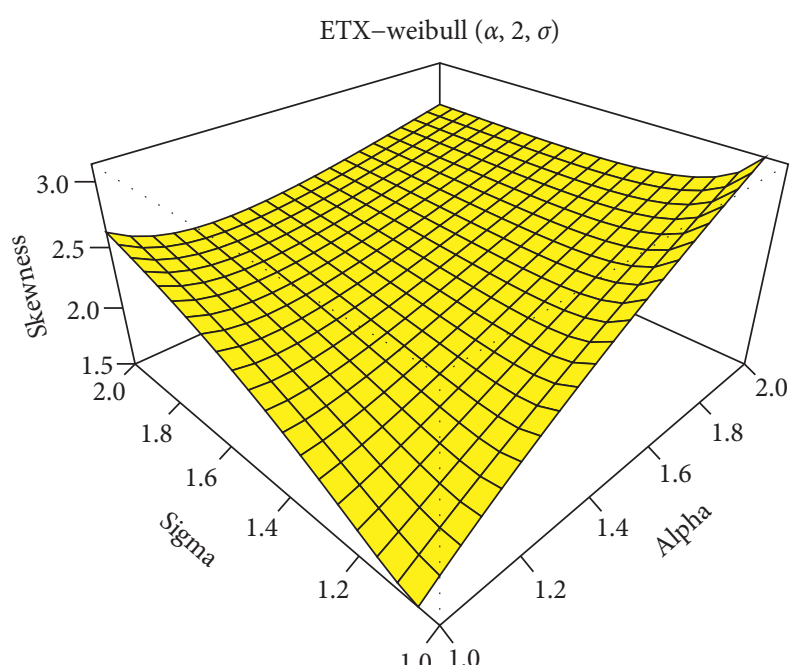

(a)

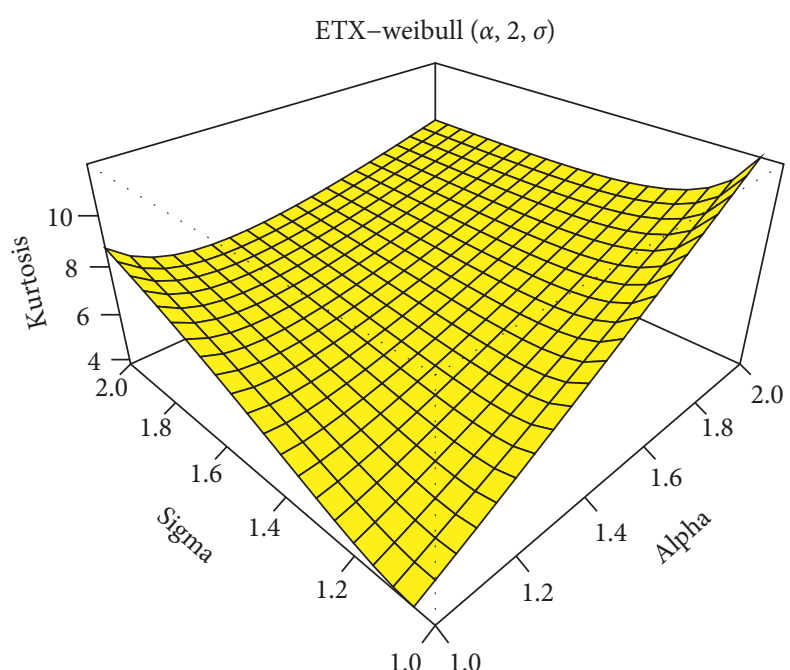

(b)

Figure 2: Plots of the skewness and kurtosis of the ETX-Weibull model.

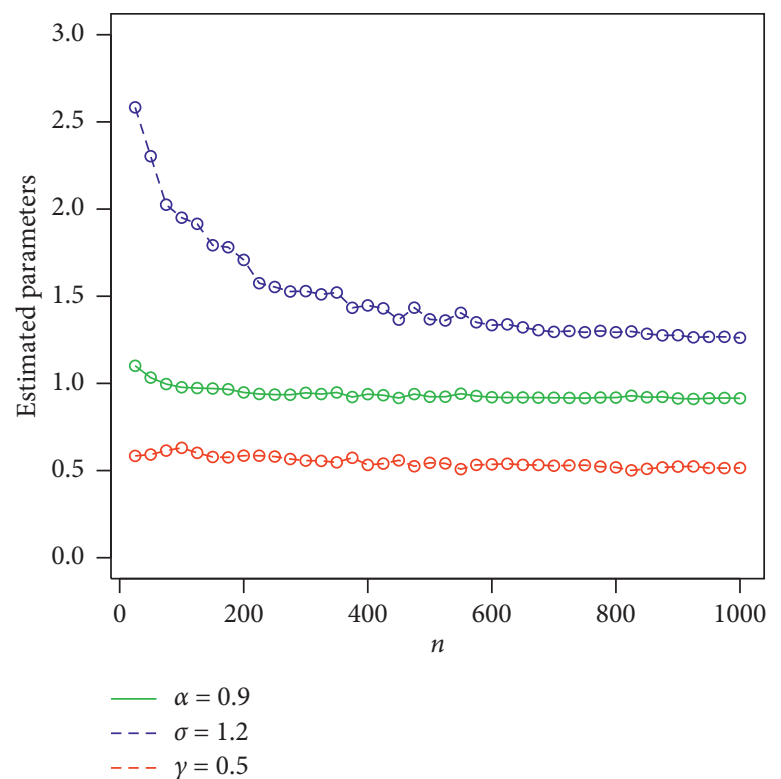

(a)

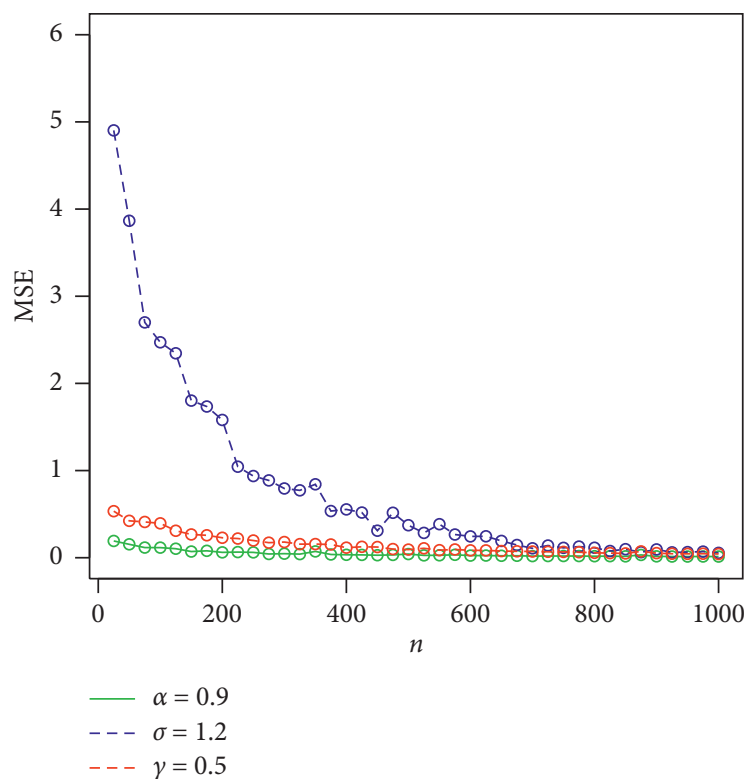

(b)

Figure 3: Continued. 


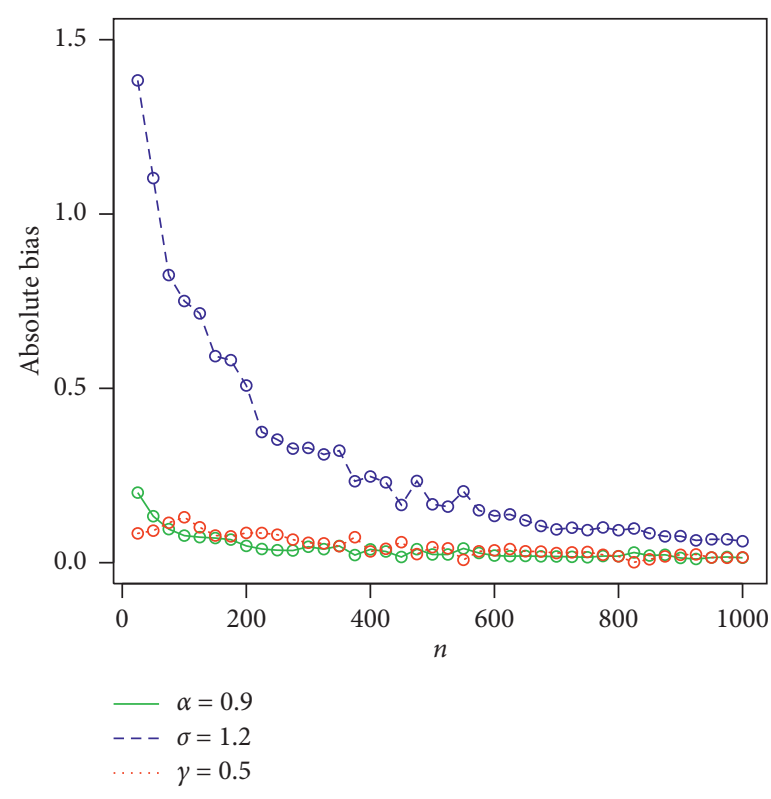

(c)

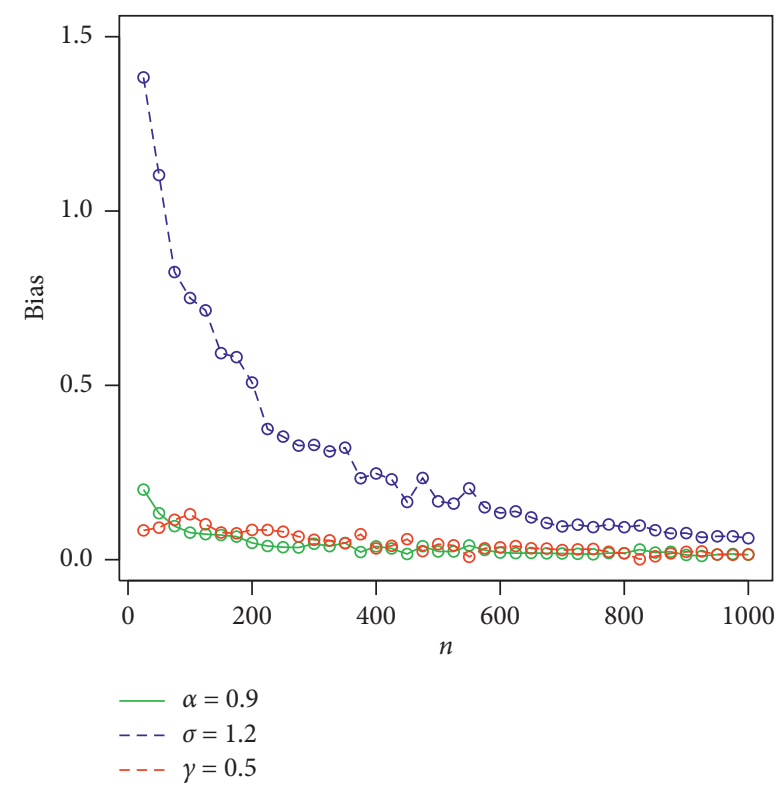

(d)

Figure 3: Plots of the estimated parameters, MSEs, absolute biases, and biases for $\alpha=0.9, \sigma=1.2=$, and $\gamma=0.5$. (a) Plot of estimated parameters versus $n$. (b) Plot of MSE versus $n$. (c) Plot of absolute bias versus $n$. (d) Plot of bias versus $n$.

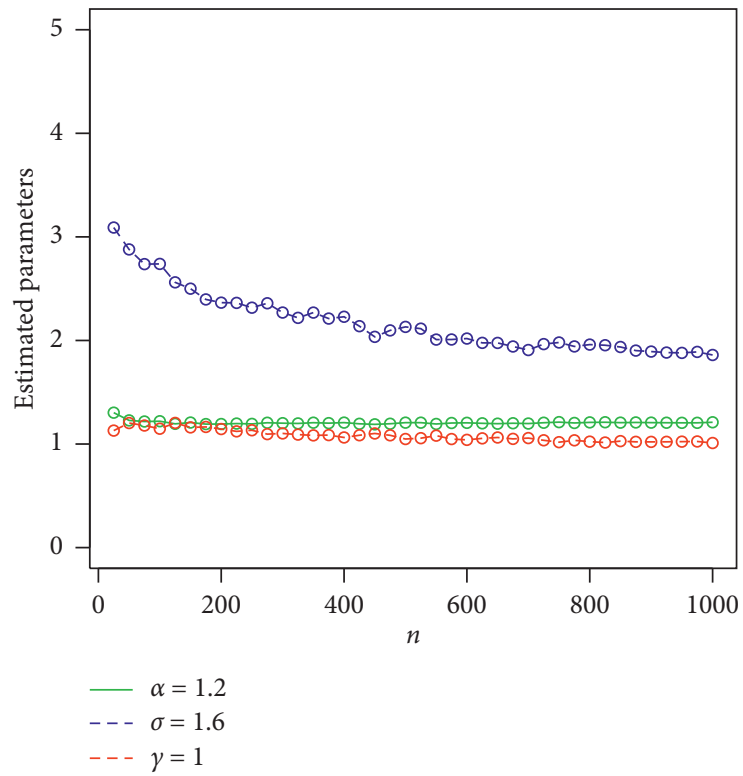

(a)

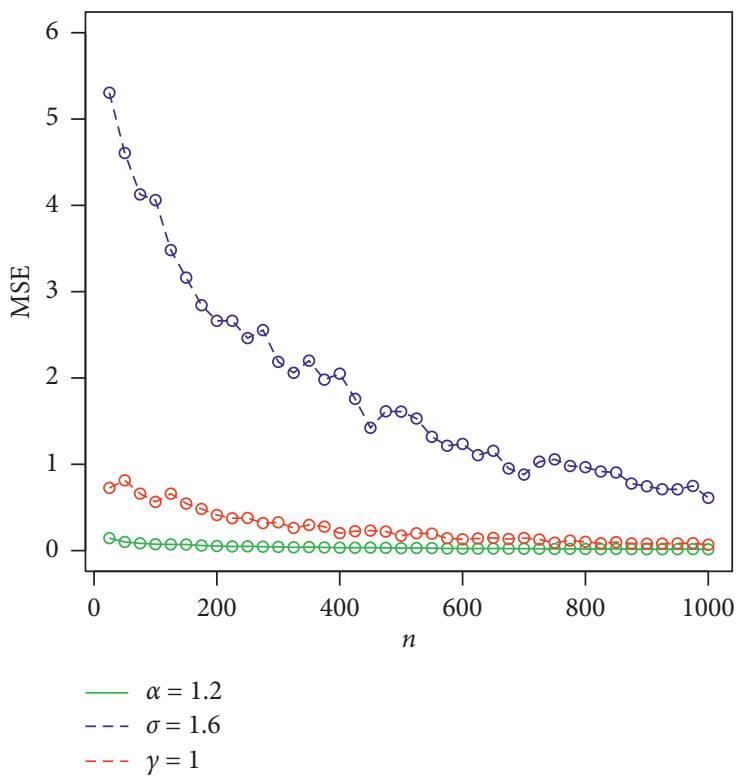

(b)

Figure 4: Continued. 


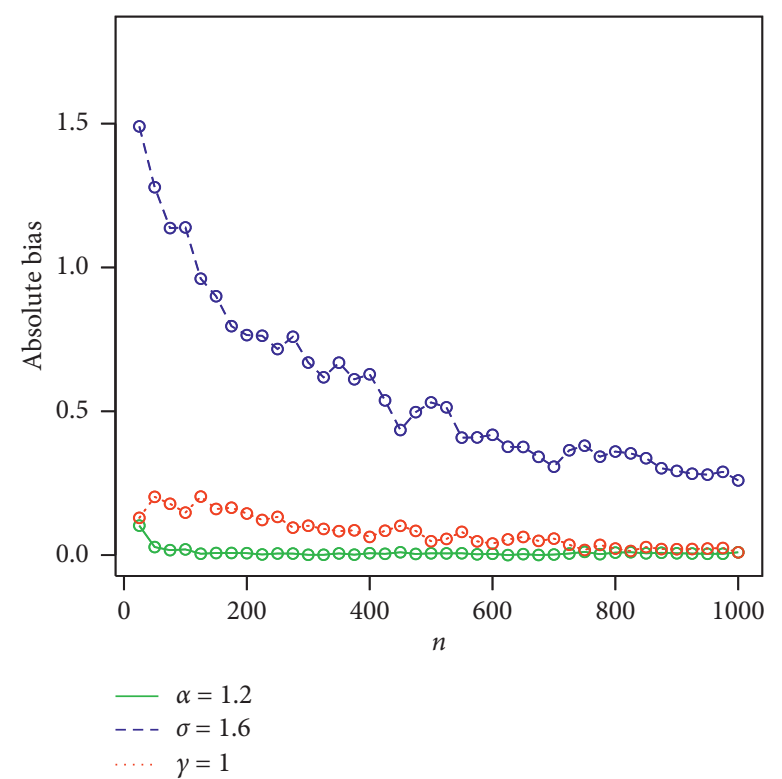

(c)

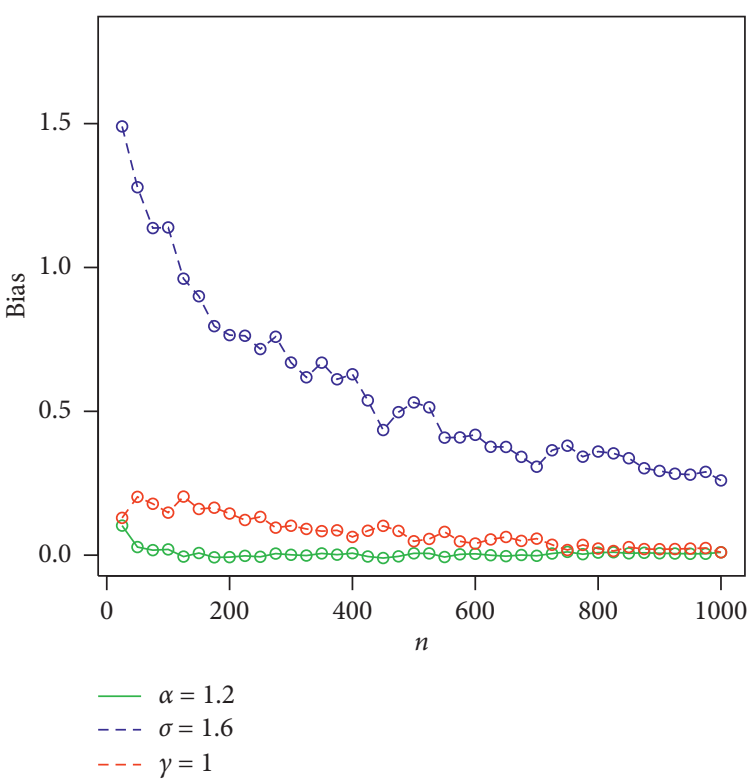

(d)

Figure 4: Plots of the estimated parameters, MSEs, absolute biases, and biases for $\alpha=1.2, \sigma=1.6=$, and $\gamma=1$. (a) Plot of estimated parameters versus $n$. (b) Plot of MSE versus $n$. (c) Plot of absolute bias versus $n$. (d) Plot of bias versus $n$.

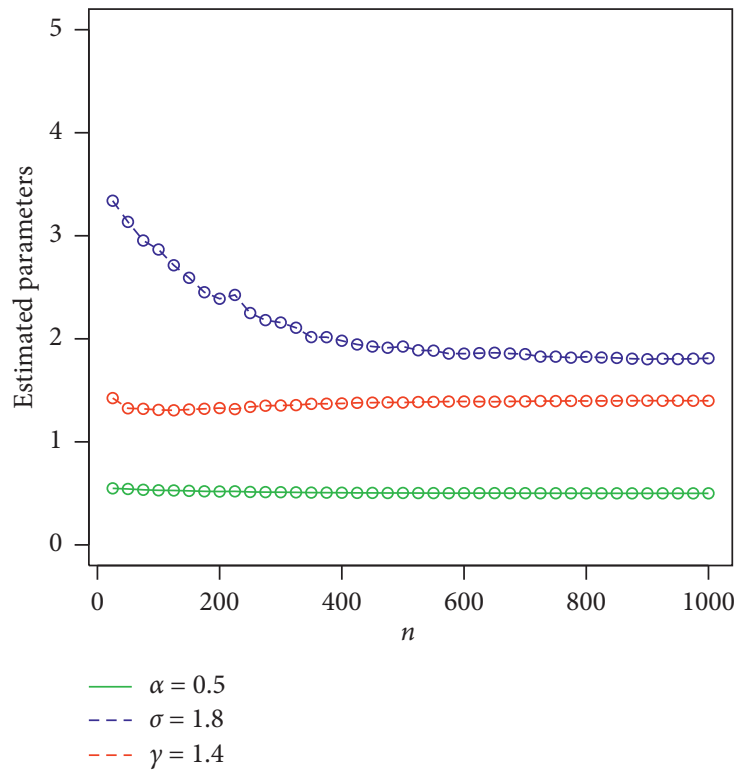

(a)

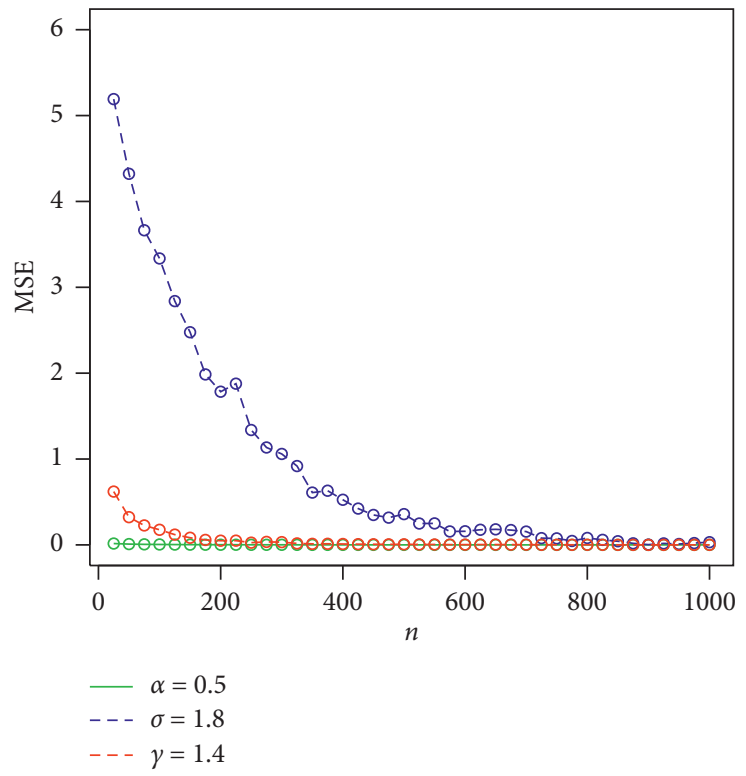

(b)

Figure 5: Continued. 


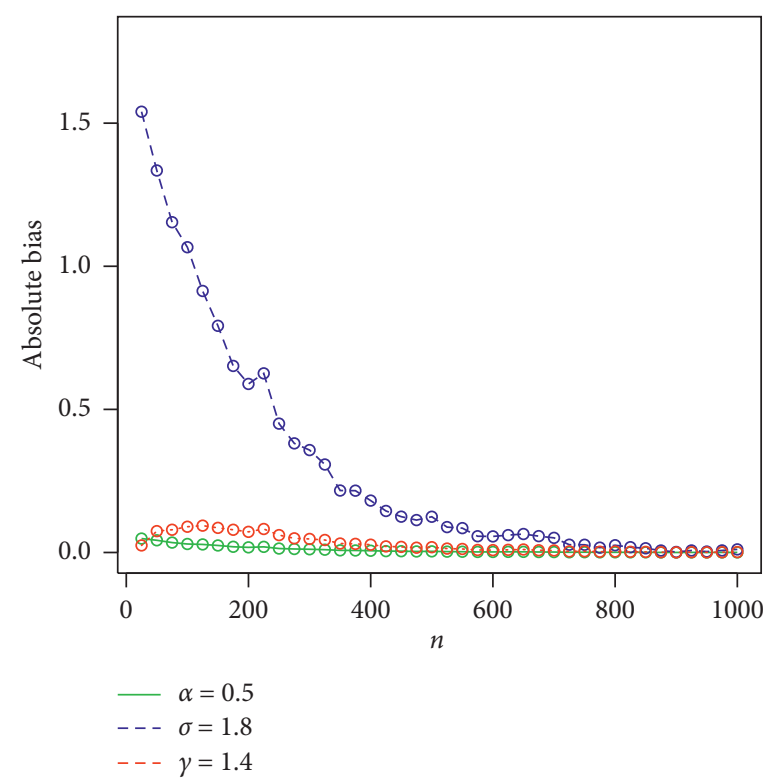

(c)

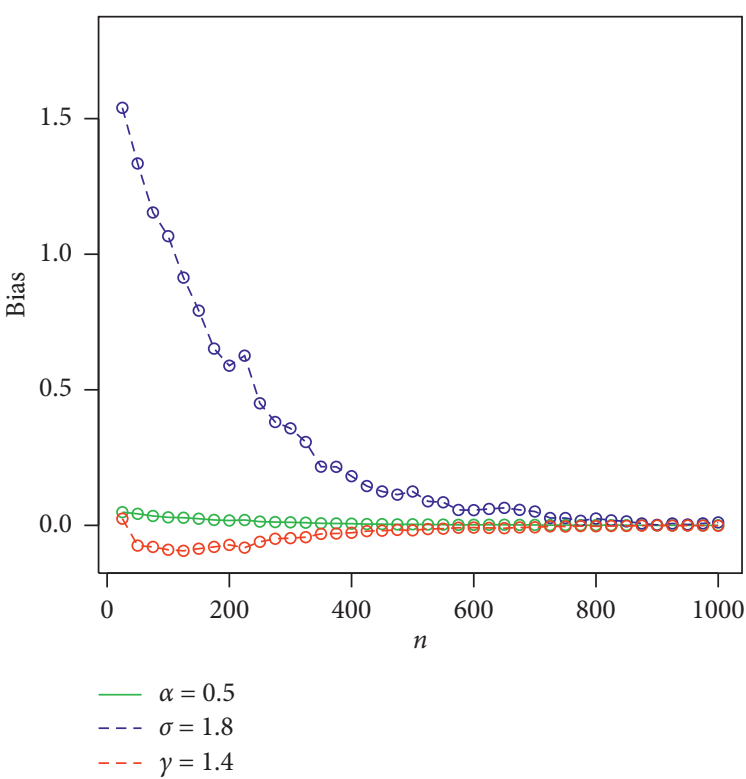

(d)

Figure 5: Plots of the estimated parameters, MSEs, absolute biases, and biases for $\alpha=0.5, \sigma=1.8=$, and $\gamma=1.4$. (a) Plot of estimated parameters versus $n$. (b) Plot of MSE versus $n$. (c) Plot of absolute bias versus $n$. (d) Plot of bias versus $n$.

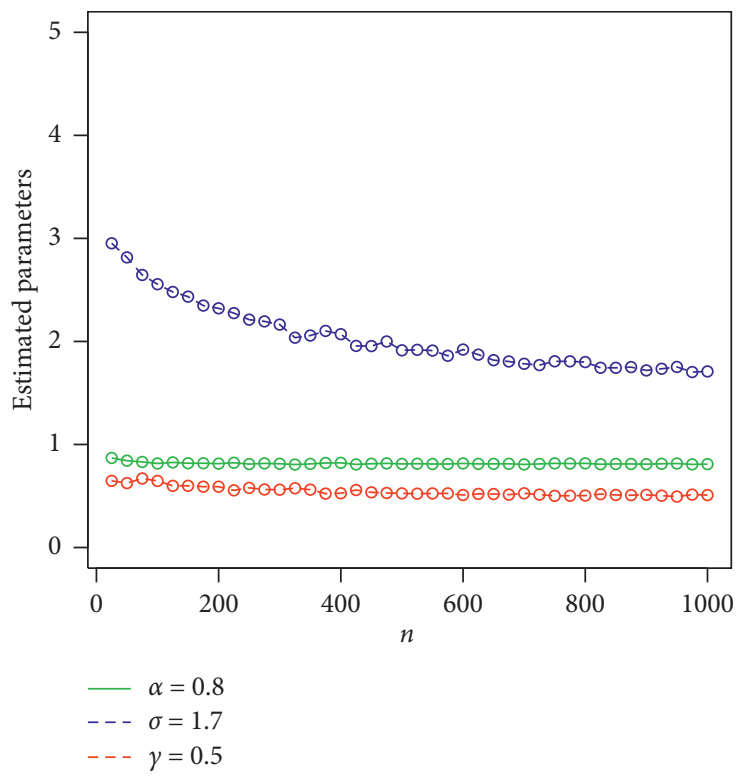

(a)

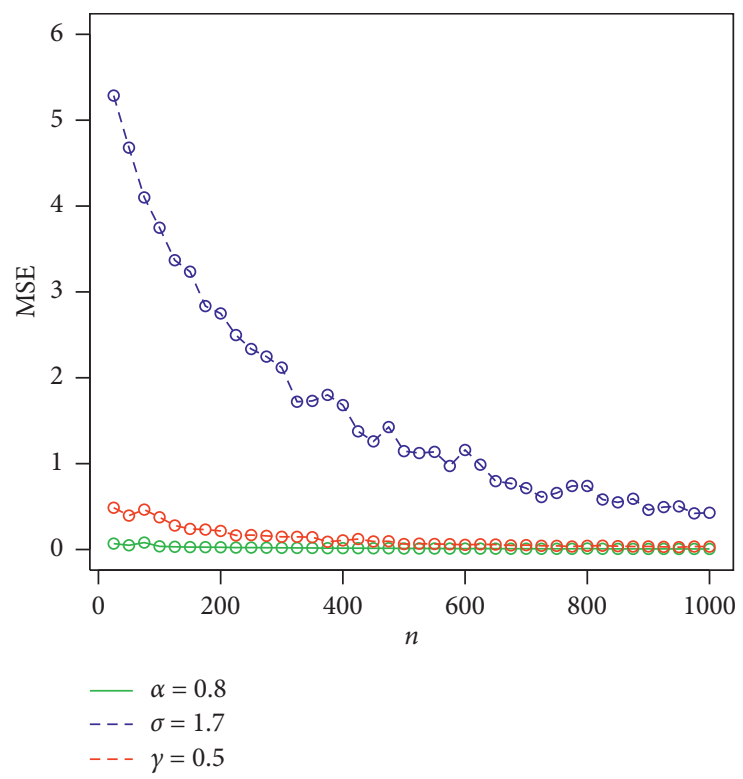

(b)

FIgURE 6: Continued. 


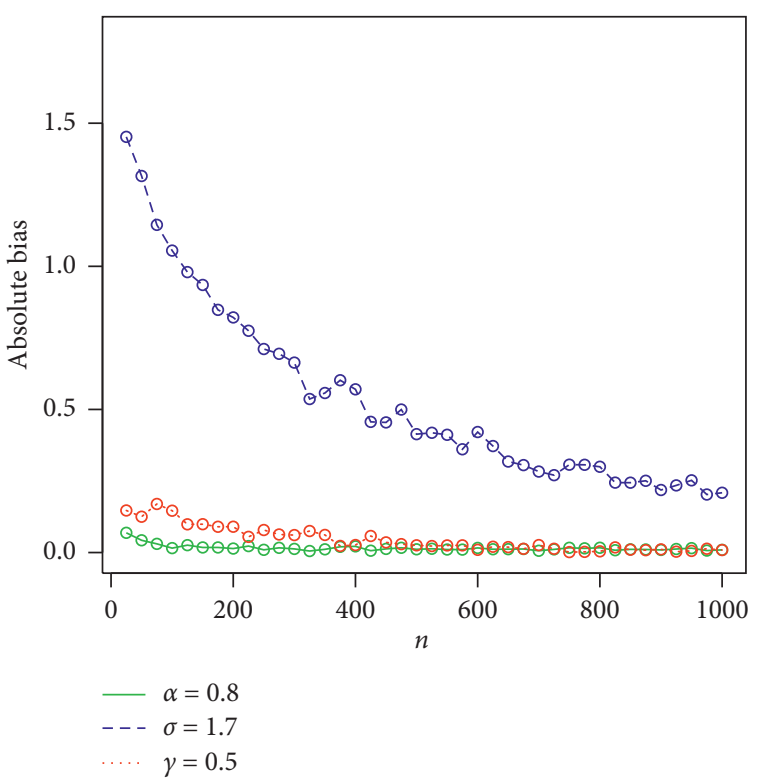

(c)

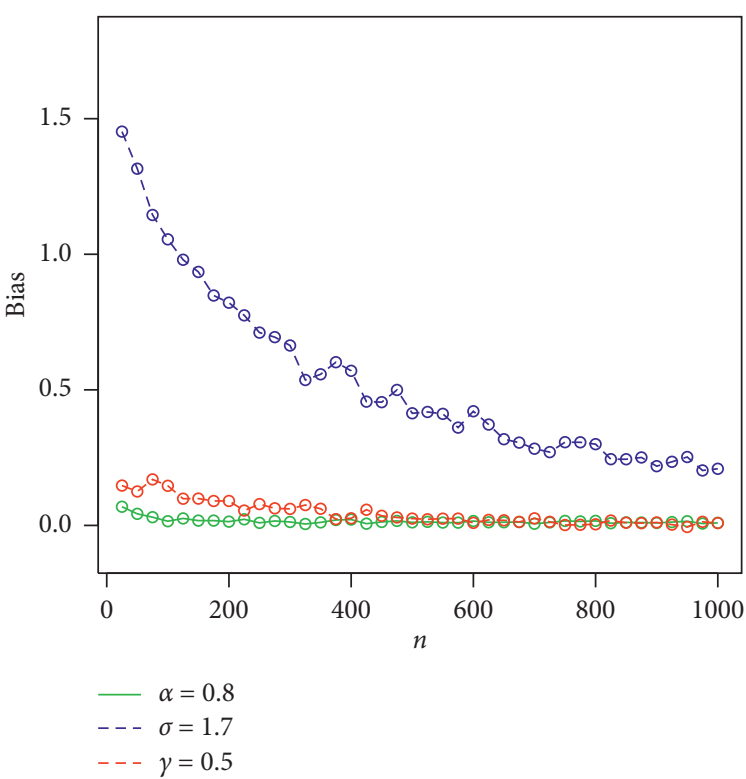

(d)

Figure 6: Plots of the estimated parameters, MSEs, absolute biases, and biases for $\alpha=0.8, \sigma=1.7=$, and $\gamma=0.5$. (a) Plot of estimated parameters versus $n$. (b) Plot of MSE versus $n$. (c) Plot of absolute bias versus $n$. (d) Plot of bias versus $n$.

(iv) As the sample size $n$ increases, the estimated biases decrease

The numerical results presented through plots reveal the consistency property of the MLEs.

\section{Actuarial Measures}

One of the most important tasks of actuarial sciences institutions is to evaluate the exposure to market risk in a portfolio of instruments, which arise from changes in underlying variables such as prices of equity, interest rates, or exchange rates. In this section, we calculate $\mathrm{VaR}$ and $\mathrm{TVaR}$ for the proposed distribution.

5.1. Value at Risk. In the context of actuarial sciences, the measure VaR is widely used by practitioners as standard financial market risk. It is also known as the quantile risk measure or quantile premium principle. The VaR is always specified with a given degree of confidence, say $q$ (typically $90 \%, 95 \%$ or, $99 \%$ ), and represents the percentage loss in the portfolio value that will be equal to or exceed only $X$ percent of the time. VaR of a random variable $X$ is the $q^{\text {th }}$ quantile of its cdf; see the work of Artzner (1999). If $X$ has cdf (6), then

$$
x_{q}=F^{-1}(t)
$$

where $t$ is the solution of the equation $t(\sigma+q-1)-q \sigma=0$.

5.2. Tail Value at Risk. Another important measure is TVaR, also known as conditional tail expectation (CTE) or tail conditional expectation (TCE), which can be adopted to quantify the expected value of the loss given that an event outside a given probability level has occurred. Let $X$ follow the proposed family; then TVaR of $X$ is defined as

$$
\operatorname{TVaR}_{q}(X)=\frac{1}{1-q} \int_{\operatorname{VaR}_{q}}^{\infty} x g(x ; \sigma, \xi) \mathrm{d} x .
$$

Using (10) in (27), we have

$$
\operatorname{TVaR}_{q}(X)=\frac{1}{1-q} \sum_{n=1}^{\infty} \sum_{i=0}^{n-1}(-1)^{i+n-1}\left(\begin{array}{c}
n-1 \\
i
\end{array}\right) \frac{n \sigma}{(i+1)^{(1 / \alpha)+1} \gamma^{1 / \alpha}(\sigma-1)^{n}} \times \Gamma\left(\frac{1}{\alpha}+1, \gamma(i+1)\left(\operatorname{VaR}_{q}\right)^{\alpha}\right) .
$$

5.3. Simulation Study of Risk Measures. In this subsection, we provide a numerical study of the risk measures for the two parameters traditional Weibull and ETX-Weibull models for different sets of parameters. The process is described as follows: (i) Random samples of size $n=150$ are generated from the Weibull and ETX-Weibull models and parameters have been estimated via the maximum likelihood method 
(ii) 1000 repetitions are made to calculate the $\mathrm{VaR}$ and TVaR for these distributions

A model with higher values of the risk measures (VaR and TVaR) is said to have heavier tails. The simulation results provided in Tables 1 and 2 show that the proposed model has higher values of the risk measures than the traditional Weibull distribution. In the light of the results provided in Tables 1 and 2 as well as in Figures 7 and 8, we can easily detect that ETX-Weibull distribution has heavier tails than the Weibull distribution. The simulation study of the $\mathrm{VaR}$ and $\mathrm{TVaR}$ is a prominent approach to determine the heavy-tailed distributions empirically.

In support of Table 1, the graphs for the VaR and TVaR of the Weibull and ETX-Weibull distributions are sketched in Figure 7.

In support of Table 2, the graphs for the VaR and TVaR of the Weibull and ETX-Weibull distributions are sketched in Figure 8.

\section{An Application and Numerical Computation of VaR and TVaR}

In this section, we illustrate the ETX-Weibull model by analyzing automobile insurance claim data to show how the proposed method works in practice. Furthermore, we calculate the actual measures of the Weibull and ETX-Weibull distributions using the real data set.

6.1. Automobile Insurance Claim Data. In this subsection, we illustrate the proposed model by analyzing a heavy-tailed real data set representing the automobile insurance claim data. The data set can be found at http:// Auto_Insurance_Claims_Sample.csv. This data has also been studied by [25]. These data are used for comparison of the ETX-Weibull distribution with the other heavy-tailed distributions. For the comparison purposes, we consider some well-known (i) two-parameter models such as Lomax, Burr-XII (BX-II), gamma, and log-normal distributions, (ii) three-parameter models such as modified Weibull (MW) and Weibull-Claim (W-Claim) distributions, and (iii) a four-parameter distribution such as the new Weibull BurrXII (NWBX-II) distribution. The distribution functions of the competitive distributions are as follows.

(i) Lomax distribution:

$$
f(x)=\alpha \gamma(1+\gamma x)^{-\alpha-1}, \quad x, \alpha, \gamma>0
$$

(ii) BX-II distribution:

$$
f(x)=\alpha \gamma x^{\gamma-1}\left(1+x^{\gamma}\right)^{-\alpha-1}, \quad x, \alpha, \gamma>0 .
$$

(iii) Log-normal distribution:

$$
f(x)=\frac{1}{x \sigma \sqrt{2 \pi}} e^{-\frac{(\ln x-\mu)^{2}}{2 \sigma^{2}}}, \quad x, \sigma>0, \mu \in \mathbb{R} .
$$

(iv) Gamma distribution:

$$
f(x)=\frac{\gamma^{\alpha}}{\Gamma(\alpha)} x^{\alpha-1} e^{-\gamma x}, \quad x, \alpha, \gamma>0
$$

(v) W-Claim distribution:

$$
f(x)=\frac{\alpha \gamma \sigma x^{\alpha-1} e^{-\gamma x^{\alpha}}\left(1-e^{-\gamma x^{\alpha}}\right)\left(2-(1-\sigma)\left(1-e^{-\gamma x^{\alpha}}\right)\right)}{\left(1-(1-\sigma)\left(1-e^{-\gamma x^{\alpha}}\right)\right)^{2}}, \quad x, \alpha, \gamma, \sigma>0 .
$$

(vi) MW distribution:

$$
f(x)=\left(\sigma+\alpha \gamma x^{\alpha-1}\right) e^{-\sigma x-\gamma x^{\alpha}}, \quad x, \alpha, \gamma, \sigma>0 .
$$

(vii) NWBX-II distribution:

$$
f(x)=\frac{\alpha \gamma c k x^{c-1} \exp \left(-\gamma\left(k \log \left(1+x^{c}\right)\right)^{\alpha}\right)}{\left(1+x^{c}\right)\left(k \log \left(1+x^{c}\right)\right)^{1-\alpha}}, \quad x \geq 0, c, k, \alpha, \gamma>0 .
$$

To decide about the goodness of fit among the proposed and other competitive distributions, we consider certain analytical measures. These measures include Akaike information criterion (AIC), Bayesian information criterion (BIC), Hannan-Quinn information criterion (HQIC), and consistent Akaike information criterion (CAIC). These measures are given as follows.

(i) AIC is given by

$$
\mathrm{AIC}=2 k-2 \ell .
$$

(ii) BIC is given by

$$
\mathrm{BIC}=k \log (n)-2 \ell .
$$


TABLE 1: Simulation results of VaR and TVaR of the Weibull and ETX-Weibull distributions for $n=150$.

\begin{tabular}{|c|c|c|c|c|}
\hline Dist. & Par. & Level of significance & $\mathrm{VaR}$ & TVaR \\
\hline \multirow{8}{*}{ Weibull } & \multirow{8}{*}{$\begin{array}{l}\alpha=0.800 \\
\gamma=0.900\end{array}$} & 0.700 & 1.434 & 3.140 \\
\hline & & 0.750 & 1.711 & 3.455 \\
\hline & & 0.800 & 2.062 & 3.849 \\
\hline & & 0.850 & 2.532 & 4.371 \\
\hline & & 0.900 & 3.226 & 5.129 \\
\hline & & 0.950 & 4.483 & 6.483 \\
\hline & & 0.975 & 5.815 & 7.900 \\
\hline & & 0.999 & 12.739 & 15.120 \\
\hline \multirow{8}{*}{ ETX-Weibull } & \multirow{8}{*}{$\begin{array}{l}\alpha=0.800 \\
\sigma=1.600 \\
\gamma=0.900\end{array}$} & 0.700 & 2.322 & 4.681 \\
\hline & & 0.750 & 2.724 & 5.114 \\
\hline & & 0.800 & 3.226 & 5.651 \\
\hline & & 0.850 & 3.885 & 6.355 \\
\hline & & 0.900 & 4.839 & 7.369 \\
\hline & & 0.950 & 6.530 & 9.156 \\
\hline & & 0.975 & 8.293 & 11.008 \\
\hline & & 0.999 & 17.267 & 20.317 \\
\hline
\end{tabular}

TABLE 2: Simulation results of VaR and TVaR of the Weibull and ETX-Weibull distributions for $n=150$.

\begin{tabular}{|c|c|c|c|c|}
\hline Dist. & Par. & Level of significance & $\mathrm{VaR}$ & TVaR \\
\hline \multirow{8}{*}{ Weibull } & \multirow{8}{*}{$\begin{array}{l}\alpha=1.800 \\
\gamma=1.500\end{array}$} & 0.700 & 0.879 & 1.205 \\
\hline & & 0.750 & 0.951 & 1.263 \\
\hline & & 0.800 & 1.033 & 1.331 \\
\hline & & 0.850 & 1.132 & 1.415 \\
\hline & & 0.900 & 1.261 & 1.526 \\
\hline & & 0.950 & 1.459 & 1.701 \\
\hline & & 0.975 & 1.638 & 1.863 \\
\hline & & 0.999 & 2.321 & 2.498 \\
\hline \multirow{8}{*}{ ETX-Weibull } & \multirow{8}{*}{$\begin{array}{l}\alpha=1.800 \\
\sigma=2.500 \\
\gamma=1.500\end{array}$} & 0.700 & 1.053 & 1.426 \\
\hline & & 0.750 & 1.135 & 1.493 \\
\hline & & 0.800 & 1.230 & 1.571 \\
\hline & & 0.850 & 1.343 & 1.666 \\
\hline & & 0.900 & 1.490 & 1.793 \\
\hline & & 0.950 & 1.716 & 1.994 \\
\hline & & 0.975 & 1.921 & 2.179 \\
\hline & & 0.999 & 2.708 & 2.914 \\
\hline
\end{tabular}

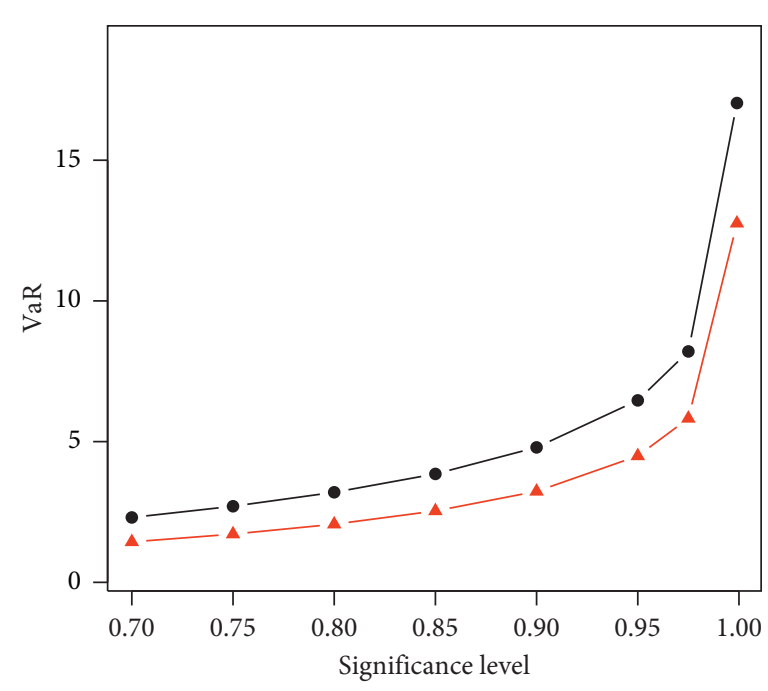

A Weibull

- ETX-weibull

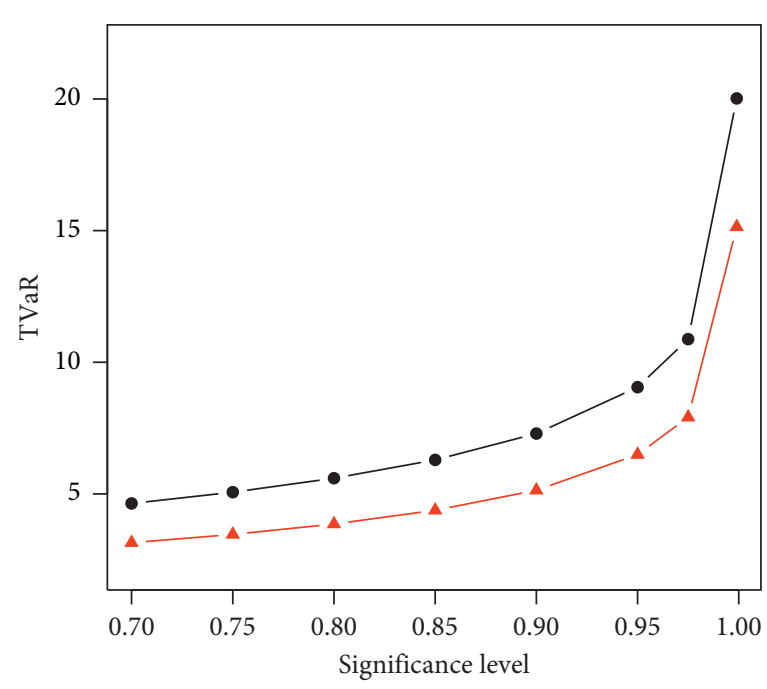

A Weibull

- ETX-weibull

(a)

(b)

FIgURE 7: Plots for the VaR and TVaR of the Weibull and ETX-Weibull distributions. 


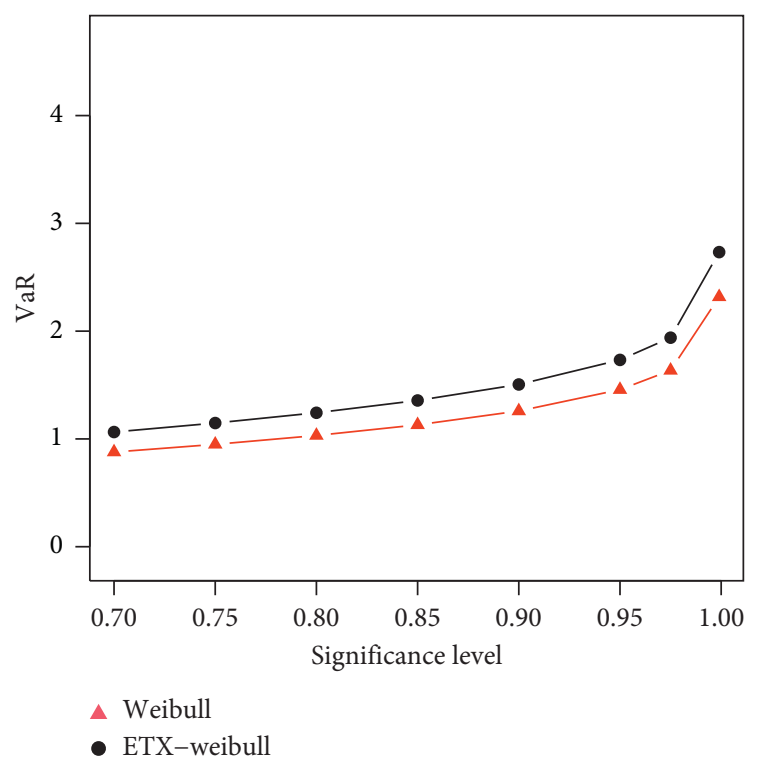

(a)

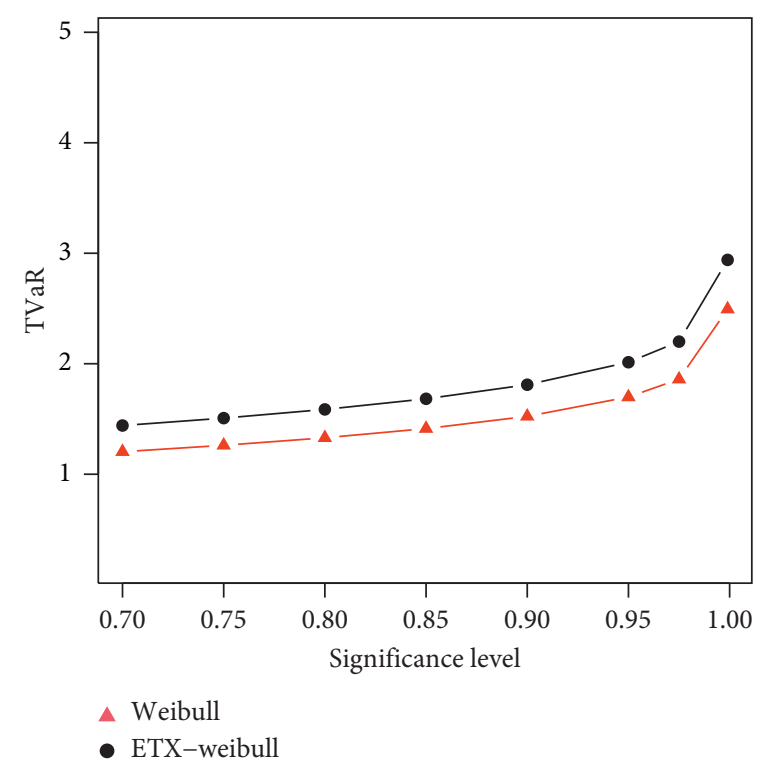

(b)

FIgure 8: Plots for the VaR and TVaR of the Weibull and ETX-Weibull distributions.

(iii) HQIC is given by

$$
\mathrm{HQIC}=2 k \log (\log (n))-2 \ell
$$

(iv) CAIC is given by

$$
\mathrm{CAIC}=\frac{2 n k}{n-k-1}-2 \ell,
$$

where $\ell$ denotes the log-likelihood function evaluated at the MLEs, $k$ is the number of model parameters, and $n$ is the sample size. The maximum likelihood estimates of the model parameters are provided in Table 3, whereas the analytical results are presented in Table 4.

From Table 4, it is clear that the proposed distribution has lower values of these measures than the other models applied in comparison. The fitted pdf and cdf plots of the proposed model for the analyzed data set are plotted in Figure 9, whereas the Kaplan-Meier survival plot of the proposed distribution and box plot of the data set are sketched in Figure 10. From Figure 9, it is clear that the proposed model fits the estimated pdf and cdf plots very closely. From Figure 10, we can easily detect that the data set has a heavy tail skewed to the right (box plot) and the ETXWeibull distribution fits the Kaplan-Meier survival plot very closely.

6.2. Computation of VaR and TVaR Using Automobile Insurance Claim Data. In this subsection, we compute the VaR and TVaR measures of the Weibull and the ETX-Weibull distributions using the estimated values of the parameters of the data set analyzed in subsection 6.1. The numerical results are reported in Table 5.
TABLE 3: The estimated values of the parameters with standard errors (in parenthesis) of the fitted distributions.

\begin{tabular}{lcccccc}
\hline Dist. & $\widehat{\alpha}$ & $\hat{\gamma}$ & $\widehat{\sigma}$ & $\widehat{c}$ & $\hat{k}$ & $\widehat{\mu}$ \\
\hline ETX-Weibull & 0.416 & 1.049 & 1.025 & & & \\
W-Claim & 1.074 & 0.013 & 1.207 & & & \\
Log-normal & & & 1.058 & & & 0.891 \\
Gamma & 1.207 & 0.098 & & & & \\
NWBX-II & 1.079 & 2.083 & & 1.513 & 0.481 & \\
Weibull & 1.266 & 0.004 & & & & \\
MW & 0.098 & 0.001 & 0.012 & & & \\
Lomax & 0.850 & 8.003 & & & & \\
BX-II & 1.084 & 2.028 & & & & \\
\hline
\end{tabular}

TABLE 4: The estimated values of the parameters with standard errors (in parenthesis) of the fitted distributions.

\begin{tabular}{lcccc}
\hline Dist. & AIC & BIC & CIAC & HQIC \\
\hline ETX-Weibull & 95341.576 & 95345.920 & 95342.324 & 95342.934 \\
W-Claim & 95415.530 & 95436.807 & 95421.329 & 95422.815 \\
Log-normal & 95527.789 & 95539.644 & 95532.409 & 95535.307 \\
Gamma & 95632.908 & 95652.389 & 95642.739 & 95645.219 \\
NWBX-II & 95937.919 & 95966.306 & 95943.180 & 95947.579 \\
Weibull & 96832.249 & 96846.428 & 96839.604 & 96837.089 \\
MW & 98359.409 & 98380.801 & 98365.911 & 98366.769 \\
Lomax & 104817.776 & 104832.098 & 104821.829 & 104822.601 \\
BX-II & 119931.802 & 119946.020 & 119939.712 & 119936.701 \\
\hline
\end{tabular}

As we have mentioned earlier, the distribution with higher values of risk measures is said to possess heavier tails. From the numerical results for the $\mathrm{VaR}$ and $\mathrm{TVaR}$ of the Weibull and ETX-Weibull distributions provided in Table 5, it is clear that the proposed distribution has a heavier tail than the Weibull distribution and can be used as a good candidate model for modeling heavy-tailed insurance data sets. 


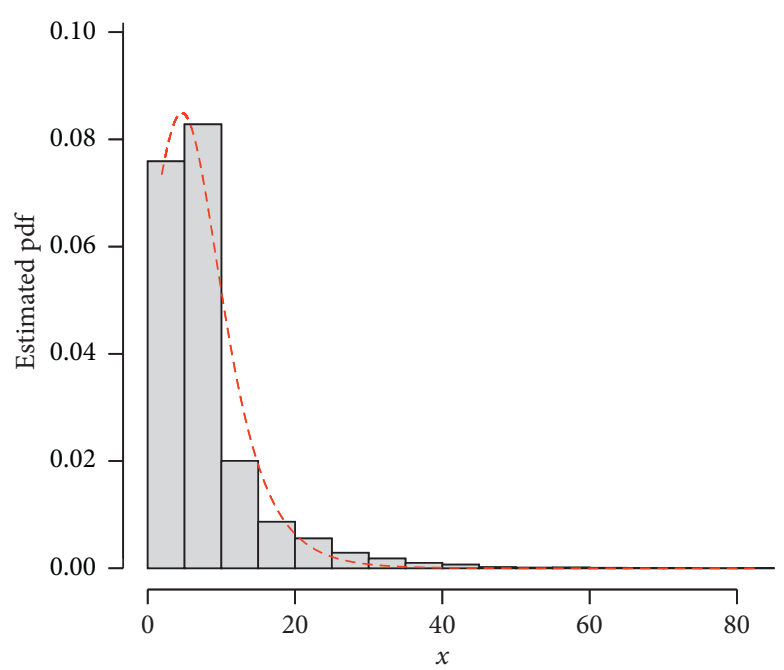

(a)

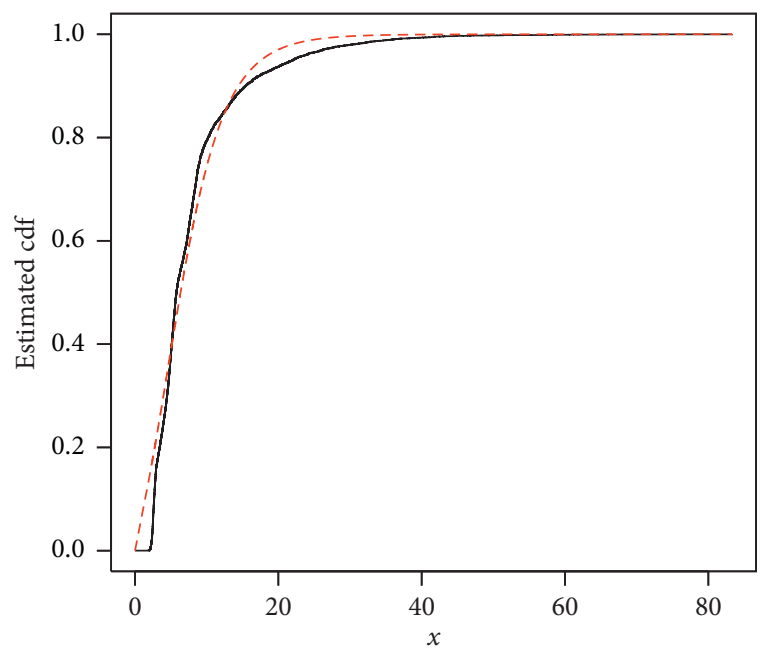

(b)

FIgURE 9: Fitted pdf and cdf of the ETX-Weibull distribution for the automobile insurance claim data set.

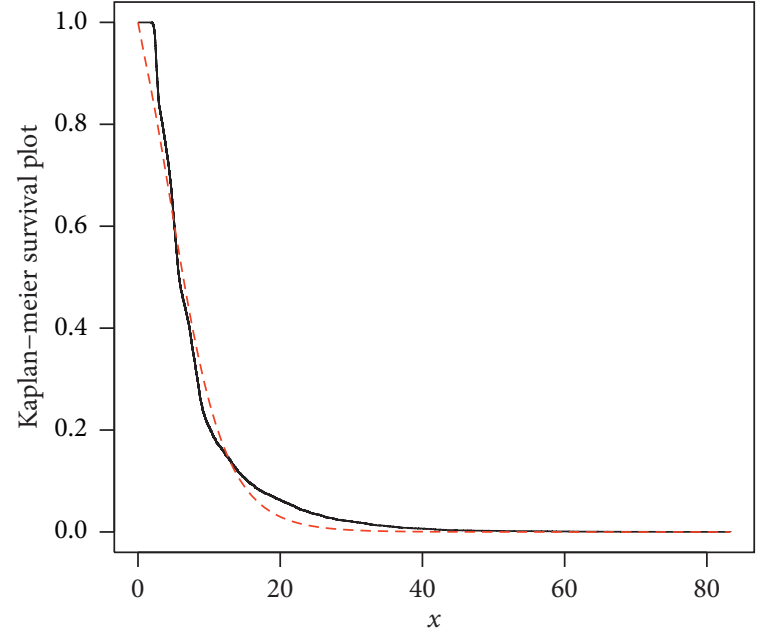

(a)

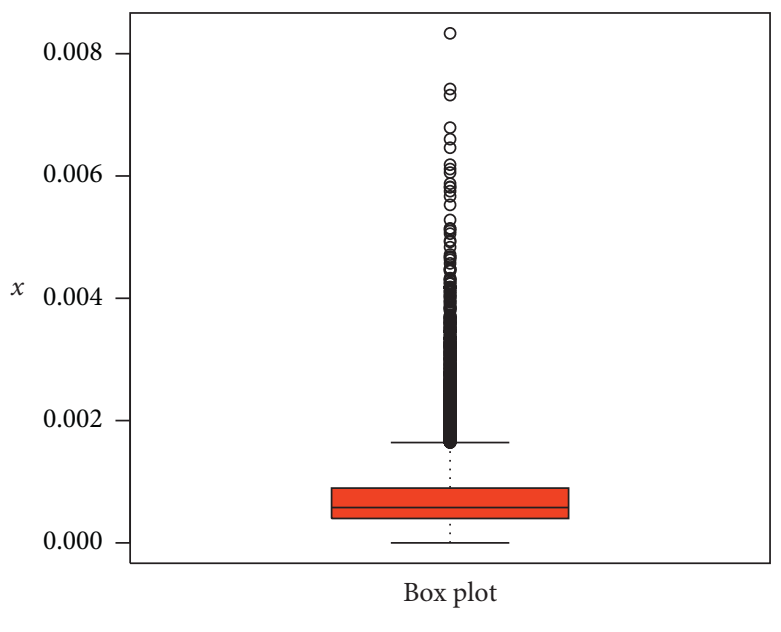

(b)

FIgURE 10: Kaplan-Meier survival plot of the ETX-Weibull distribution and box plot of the automobile insurance claim data set.

TABLE 5: The estimated values of the parameters with standard errors (in parenthesis) of the fitted distributions.

\begin{tabular}{lcccc}
\hline Dist. & Par. & Level of significance & VaR & TVaR \\
\hline & & 0.700 & 98.118 & 155.719 \\
Weibull & $\widehat{\alpha}=1.261$ & 0.800 & 123.320 & 163.507 \\
& $\widehat{\gamma}=0.004$ & 0.900 & 282.247 & 178.579 \\
& & 0.999 & 197.035 & 328.704 \\
ETX-Weibull & $\widehat{\alpha}=0.416$ & 0.700 & 257.007 & 327.972 \\
& $\widehat{\gamma}=1.049$ & 0.800 & 365.878 & 385.873 \\
& $\widehat{\sigma}=1.025$ & 0.900 & 830.701 & 493.883 \\
\hline
\end{tabular}




\section{Concluding Remarks}

In this article, a new heavy-tailed family of claim distributions is proposed. For illustrative purposes, a special model of the proposed family is considered, called ETXWeibull distribution. The ETX-Weibull model is very flexible in modeling heavy-tailed data. Some mathematical properties of the proposed family are derived, and maximum likelihood estimators of the model parameters are obtained. A comprehensive simulation study is presented to explore the behavior of these estimators. Actuarial measures of the ETX-Weibull model are also calculated, and a simulation study is conducted to show the usefulness of the proposed method in actuarial sciences. The simulation study of the actuarial measures shows that the proposed model possesses heavier tails. Finally, an automobile insurance claim data set is analyzed, and the comparison of the proposed model is made with other well-known competitors. The application shows that the ETX-Weibull distribution may be a good candidate for modeling heavy-tailed insurance data sets.

\section{Appendix}

\section{R Code for the Simulation Study}

In the following R-code, a is used for $\alpha, \mathrm{s}$ is used for $\sigma$, and $\mathrm{g}$ is used for $\gamma$.

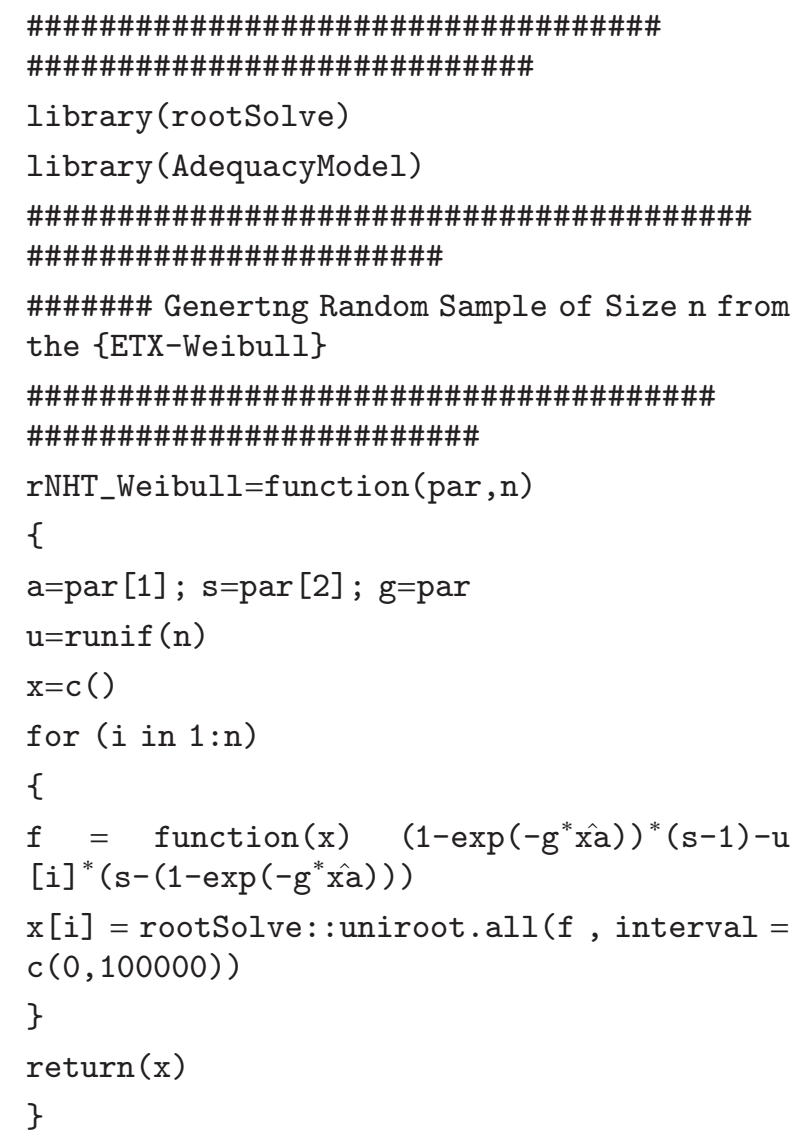

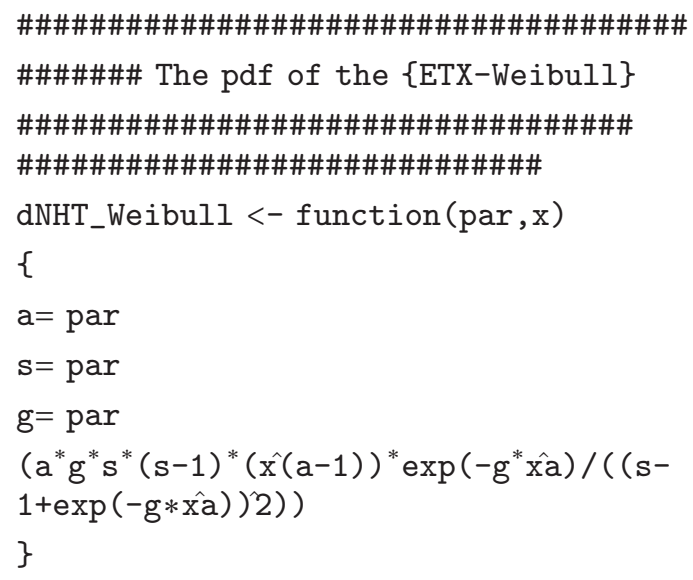


\}

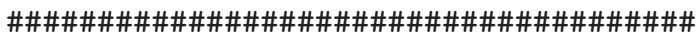

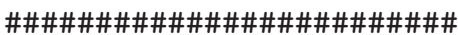

\#\#\#\#\#\# The Function for the simulation stuyd of the $\{$ ETX-Weibull $\}$

\#\#\#\#\#\#\#\#\#\#\#\#\#\#\#\#\#\#\#\#\#\#\#\#\#\#\#\#\#\#\#\#\#\#\#\#\# \#\#\#\#\#\#\#\#\#\#\#\#\#\#\#\#\#\#\#\#\#

$\operatorname{par}<-c(0.9,1.2,0.5) ; \mathrm{a}=0.9 ; \mathrm{s}=1.2 ; \mathrm{g}=0.5$

n_replicas $=1000$

matriz_par <- matrix $(0,40,3)$

matriz_bias<- matrix $(0,40,3)$

matriz_MSE <- matrix $(0,40,3)$

matriz_std <- matrix $(0,40,3)$

colnames (matriz_par) <- c ( a, s, g )

colnames (matriz_bias) $<-c($ a , s , g )

colnames (matriz_MSE) <- c ( a , s , g )

colnames (matriz_std) <- c ( a , s , g )

cont $=1$

$\mathrm{n}=25$

while $(\mathrm{n}<=1000)$

\{

par_mean $<-c(0,0,0)$

std_mean $<-c(0,0,0)$

bias $<-c(0,0,0)$

$\operatorname{MSE}<-c(0,0,0)$

replica $=1$

while (replica <= n_replicas)

\{

print (paste $(\mathrm{n}=, \mathrm{n}, \quad$ replica $=$, replica))

$\mathrm{x}<-r N H T$ Weibull (par, $\mathrm{n})$

Data $<-\mathrm{x}$

\#\#\#\#\#\#\#\#\#\#\#\#\#\#\#\#\#\#\#\#\#\#\#\#\#\#\#\#\#\#\#\#\#\#\#\#\#\# \#\#\#\#\#\#\#\#\#\#\#\#\#\#\#\#\#\#\#

\#\#\#\#\#\#\#\# Optimization and Generating the Simulation Results.

\#\#\#\#\#\#\#\#\#\#\#\#\#\#\#\#\#\#\#\#\#\#\#\#\#\#\#\#\#\#\#\#\#

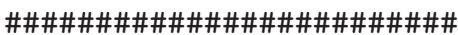

result=optim(c(a, s, g), loglikelihoodNHT_Weibull, hessian $=\mathrm{F}$,

control $=$ list $($ fnscale $=-1)$,

method $=$ L-BFGS-B , lower $=c(0.001,1.001$, $0.001)$, upper $=c(5,5,5))$

if (class(result) $\quad !=$ try-error \&\& result $\backslash \$$ convergence $==0$ )

\{

par_\{m\}ean \lt - par_\{m\}ean + result \\$par

bias = bias + (result \\$par - par)

MSE $=$ MSE + (resul \t\$par - par $)^{2} 2$

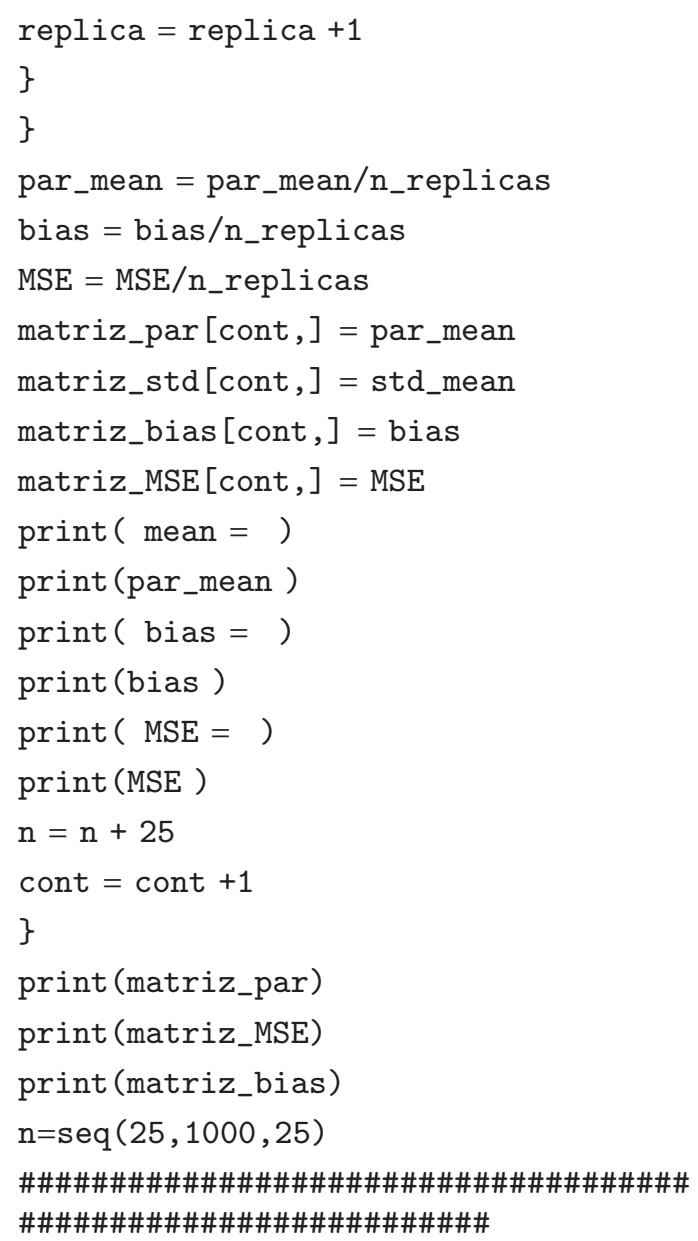

$\operatorname{plot}(n,($ matriz_par[,1]), type=“o", col=“green”, lty=1, lwd=2,xlab= "n",

$\mathrm{ylab}=$ Estimated Parameters, $\mathrm{ylim}=\mathrm{c}(0,3))$

lines(n,(matriz_par[,2]), col="blue", 1 ty $=5,1 w d=2$, type $=$ "o")

lines(n,(matriz_par[,3]), col="red", lty=8,lwd=2,type=“o") title ( Plot of Estimated Parameters vs n )

legend $(700,2.8$, legend $=c($ expression $($ paste (alpha, “=”, “0.9”)),

\#\#\#\#\#\#\#\# Plots of MSEs

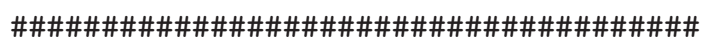
\#\#\#\#\#\#\#\#\#\#\#\#\#\#\#\#\#\#\#\#\#

plot (n,matriz_MSE $[, 1], \operatorname{col}=$ green , 1 ty $=1$, $1 \mathrm{wd}=2, \mathrm{type}=0, \mathrm{xlab}=\mathrm{n}$, 


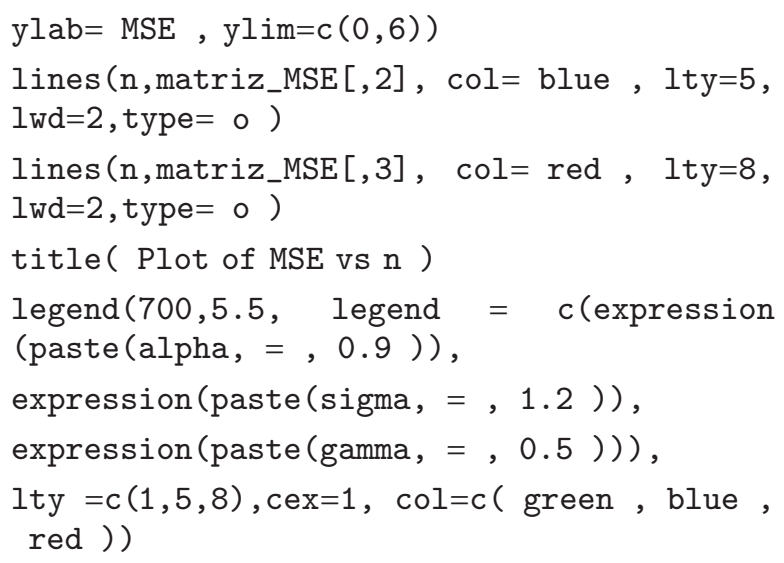

\section{Data Availability}

This work is mainly a methodological development and has been applied on secondary data related to the insurance sciences, but, if required, data will be provided.

\section{Disclosure}

This article is drafted from the Ph.D. work of the first author (Zubair Ahmad).

\section{Conflicts of Interest}

The authors declare that they have no conflicts of interest.

\section{References}

[1] M. N. Lane, "Pricing risk transfer transactions," ASTIN Bulletin, vol. 30, no. 2, pp. 259-293, 2000.

[2] K. Cooray and M. Ananda, "Modeling actuarial data with a composite lognormal-Pareto model," Scandinavian Actuarial Journal, vol. 5, pp. 321-334, 2005.

[3] S. A. Klugman, H. H. Panjer, and G. E. Willmot, "Loss models: from data to decisions," in Wiley Series in Probability and StatisticsJohn Wiley \& Sons, Hoboken, NJ, USA, 2012.

[4] Z. Ahmad, E. Mahmoudi, and M. Alizadeh, "Modelling insurance losses using a new beta power transformed family of distributions," Communications in Statistics - Simulation and Computation, vol. 49, pp. 1-22, 2020.

[5] A. J. McNeil, "Estimating the tails of loss severity distributions using extreme value theory," ASTIN Bulletin, vol. 27, no. 1, pp. 117-137, 1997.

[6] J. Beirlant, G. Matthys, and G. Dierckx, "Heavy-tailed distributions and rating," ASTIN Bulletin, vol. 31, no. 1, pp. 37-58, 2001.

[7] D. Bhati and S. Ravi, "On generalized log-Moyal distribution: a new heavy tailed size distribution," Insurance: Mathematics and Economics, vol. 79, pp. 247-259, 2018.

[8] D. P. M. Scollnik and C. Sun, "Modeling with weibull-pareto models," North American Actuarial Journal, vol. 16, no. 2, pp. 260-272, 2012.

[9] Z. Ahmad, E. Mahmoudi, G. G. Hamedani, and O. Kharazmi, "New methods to define heavy-tailed distributions with applications to insurance data," Journal of Taibah University for Science, vol. 14, no. 1, pp. 359-382, 2020.

[10] S. Nadarajah and S. A. A. Bakar, "New composite models for the Danish fire insurance data," Scandinavian Actuarial Journal, vol. 2, pp. 180-187, 2014.

[11] S. A. Abu Bakar, N. A. Hamzah, M. Maghsoudi, and S. Nadarajah, "Modeling loss data using composite models," Insurance: Mathematics and Economics, vol. 61, pp. 146-154, 2015.

[12] A. Punzo, "A new look at the inverse Gaussian distribution with applications to insurance and economic data," Journal of Applied Statistics, vol. 46, no. 7, pp. 1260-1287, 2019.

[13] A. Mazza and A. Punzo, "Modeling household income with contaminated unimodal distributions," in New Statistical Developments in Data Science, A. Petrucci, F. Racioppi, and R. Verde, Eds., Springer International Publishing, Cham, Switzerland, pp. 373-391, 2019.

[14] S. D. Tomarchio and A. Punzo, "Dichotomous unimodal compound models: application to the distribution of insurance losses," Journal of Applied Statistics, vol. 47, no. 13-15, pp. 2328-2353, 2020. 
[15] A. Punzo and L. Bagnato, "Modeling the cryptocurrency return distribution via Laplace scale mixtures," Physica A: Statistical Mechanics and Its Applications, vol. 563, p. 125354, 2021.

[16] A. Punzo, L. Bagnato, and A. Maruotti, "Compound unimodal distributions for insurance losses," Insurance: Mathematics and Economics, vol. 81, pp. 95-107, 2018.

[17] M. H. Tahir and G. M. Cordeiro, "Compounding of distributions: a survey and new generalized classes," Journal of Statistical Distributions and Applications, vol. 3, pp. 1-35, 2016.

[18] M. Bernardi, A. Maruotti, and L. Petrella, "Skew mixture models for loss distributions: a bayesian approach," Insurance: Mathematics and Economics, vol. 51, no. 3, pp. 617-623, 2012.

[19] T. Miljkovic and B. Grün, "Modeling loss data using mixtures of distributions," Insurance: Mathematics and Economics, vol. 70, pp. 387-396, 2016.

[20] A. Punzo, A. Mazza, and A. Maruotti, "Fitting insurance and economic data with outliers: a flexible approach based on finite mixtures of contaminated gamma distributions," Journal of Applied Statistics, vol. 45, no. 14, pp. 2563-2584, 2018.

[21] A. Alzaatreh, C. Lee, and F. Famoye, "A new method for generating families of continuous distributions," Metron, vol. 71, no. 1, pp. 63-79, 2013.

[22] Z. Ahmad, E. Mahmoudi, and S. Dey, "A new family of heavy tailed distributions with an application to the heavy tailed insurance loss data," Communications in Statistics - Simulation and Computation, vol. 49, pp. 1-24, 2020.

[23] E. Seneta, "Karamata's characterization theorem, feller and regular variation in probability theory," Publications de l'Institut Mathematique, vol. 71, no. 85, pp. 79-89, 2002.

[24] S. I. Resnick, "Discussion of the Danish data on large fire insurance losses," ASTIN Bulletin, vol. 27, no. 1, pp. 139-151, 1997.

[25] Z. Ahmad, E. Mahmoudi, and G. Hamedani, “A class of claim distributions: properties, characterizations and applications to insurance claim data," Communications in Statistics - Theory and Methods, vol. 49, pp. 1-26, 2020. 